\title{
Development of specialized sensory neurons engages a nuclear receptor required for functional plasticity
}

\author{
Mary Rossillo and Niels Ringstad \\ Skirball Institute of Biomolecular Medicine, Department of Cell Biology, Neuroscience Institute, New York University School \\ of Medicine, New York, New York 10016, USA
}

During development, the nervous system generates neurons that serve highly specialized roles and, accordingly, possess unique functional attributes. The chemosensory BAG neurons of $C$. elegans are striking exemplars of this. BAGs sense the respiratory gas carbon dioxide $\left(\mathrm{CO}_{2}\right)$ and, in a context-dependent manner, switch from mediating avoidance of $\mathrm{CO}_{2}$ to supporting $\mathrm{CO}_{2}$ attraction. To determine mechanisms that support the physiology and plasticity of BAG neurons, we used tandem ChIP-seq and cell targeted RNA-seq to identify gene targets of the transcription factor ETS-5, which is required for BAG development. A functional screen of ETS-5 targets revealed that NHR-6, the sole $C$. elegans NR4A-type nuclear receptor, is required for BAG-mediated avoidance of $\mathrm{CO}_{2}$ and regulates expression of a subset of BAG-specific genes. Unlike ets-5 mutants, which are defective for both attraction to and avoidance of $\mathrm{CO}_{2}, n h r-6$ mutants are fully competent for attraction. These data indicate that the remarkable ability of BAGs to adaptively assign positive or negative valence to a chemosensory stimulus requires a gene-regulatory program supported by an evolutionarily conserved type of nuclear receptor. We suggest that NHR-6 might be an example of a developmental mechanism for modular encoding of functional plasticity in the nervous system.

[Keywords: neurodevelopment; ETS transcription factor; nuclear receptor; C. elegans; chemosensation]

Supplemental material is available for this article.

Received July 3, 2020; revised version accepted September 30, 2020.

Sensory neurons possess highly specialized physiology that allows them to detect environmental cues and transduce them to the neural circuits that generate behavior. This specialized physiology is the result of genetic programs invoked during development that regulate expression of factors required for sensory transduction, for example, sensory receptors and their associated signaling pathways. Developmental programs also confer mechanisms that determine how neural activity changes in response to experience and context; i.e., functional plasticity. Understanding gene regulation that underlies sensory neuron development therefore offers the opportunity to identify the molecular mechanisms that mediate and modulate sensation.

The nematode C. elegans is a powerful model both for the study of nervous system development and for the study of sensation. Although the C. elegans nervous system contains only 302 neurons, 60 of these neurons are sensory neurons that support a wide range of sensory modalities including olfaction, gustation, mechanosensation, and thermosensation. A subset of C. elegans

Corresponding author: niels.ringstad@med.nyu.edu Article published online ahead of print. Article and publication date are online at http://www.genesdev.org/cgi/doi/10.1101/gad.342212.120. chemosensory neurons is dedicated to sensing the respiratory gases oxygen $\left(\mathrm{O}_{2}\right)$ and carbon dioxide $\left(\mathrm{CO}_{2}\right)$. The URX, AQR, and PQR sensory neurons are activated by increases in environmental $\mathrm{O}_{2}$ (Gray et al. 2004), and the BAG neurons are activated by decreases in $\mathrm{O}_{2}$ and increases in $\mathrm{CO}_{2}$ (Hallem and Sternberg 2008; Zimmer et al. 2009). Gas-sensing neurons are found in many animals and support diverse behaviors and motor programs (Ma and Ringstad 2012; Guyenet and Bayliss 2015). C. elegans live in microbe-rich environments, and several studies suggest that their gas-sensing neurons detect increases in $\mathrm{CO}_{2}$ and decreases in $\mathrm{O}_{2}$ as products of microbial respiration to control food-seeking and pathogen-avoidance behaviors (Bretscher et al. 2008; Milward et al. 2011; Brandt and Ringstad 2015).

C. elegans gas-sensing neurons all use cGMP-based chemotransduction pathways. Gas-sensing requires cyclic nucleotide-gated (CNG) ion channels encoded by the genes tax-2 and tax-4 (Hallem et al. 2011b). Upstream of

(C) 2020 Rossillo and Ringstad This article is distributed exclusively by Cold Spring Harbor Laboratory Press for the first six months after the full-issue publication date (see http://genesdev.cshlp.org/site/misc/ terms.xhtml). After six months, it is available under a Creative Commons License (Attribution-NonCommercial 4.0 International), as described at http://creativecommons.org/licenses/by-nc/4.0/. 
An NR4a receptor promotes neuronal differentiation

these channels are guanylyl cyclases that function as receptors for $\mathrm{O}_{2}$ and $\mathrm{CO}_{2}$. Gas-sensing neurons express different soluble guanylyl cyclases that are either activated by or inhibited by diatomic oxygen (Zimmer et al. 2009). The $\mathrm{CO}_{2}$ sensor, which is only expressed in BAG neurons, is the receptor-type guanylyl cyclase GCY-9 (Hallem et al. 2011 b; Smith et al. 2013). In addition to using different receptor mechanisms to sense respiratory gases, gas-sensing neurons have markedly different physiology with respect to adaptation and plasticity. URX oxygen-sensing neurons are capable of maintaining a sustained response to $\mathrm{O}_{2}$ stimuli and likely function as tonic sensors of ambient $\mathrm{O}_{2}$ (Busch et al. 2012). In contrast, BAG neuron responses to $\mathrm{CO}_{2}$ adapt within seconds (Hallem et al. 2011b). Also, BAG neurons mediate either attraction to or avoidance of $\mathrm{CO}_{2}$ depending on an individual's developmental history or prior experience. Larvae and adults grown under standard conditions avoid $\mathrm{CO}_{2}$ stimuli, but dauer larvae, which have undergone an alternative developmental path in response to crowding, are attracted to environmental $\mathrm{CO}_{2}$ (Hallem et al. 2011a). Animals that have undergone normal development but that experienced high levels of $\mathrm{CO}_{2}$ or were food-deprived also become attracted to $\mathrm{CO}_{2}$ (Guillermin et al. 2017; Rengarajan et al. 2019).

To better understand the physiology of BAG neurons, we considered the mechanisms that regulate their development and ultimately confer their unique functional attributes. Transcription factors required for expression of a BAG neuron fate have been identified and mutants for these factors have defects in BAG function. The Sox family protein EGL-13 promotes gene expression in BAGs and also in the $\mathrm{O}_{2}$-sensing URX neurons (Gramstrup Petersen et al. 2013). In contrast, the zinc finger protein EGL-46 and the ETS family transcription factor ETS-5 regulate gene expression in $\mathrm{CO}_{2}$-sensing BAG neurons but not in other gas-sensing neurons (Guillermin et al. 2011; Brandt et al. 2012; Romanos et al. 2015). Furthermore, a vertebrate homolog of ETS-5, Pet1, is required for the development of $\mathrm{CO}_{2}$-sensitive regions of the mammalian brainstem (Hendricks et al. 1999), suggesting that this transcription factor might have an ancestral role in regulating the development of gas-sensing neurons.

We sought new factors required either for the sensory function of BAGs or for their plasticity by a genomewide enumeration of ETS-5 targets followed by a functional screen. Through this approach, we identified the nuclear receptor NHR-6, whose expression is highly enriched in BAG neurons and which functions cell-autonomously to promote $\mathrm{CO}_{2}$ avoidance. Strikingly, nhr-6 mutants are fully competent for attraction to $\mathrm{CO}_{2}$ under conditions that switch the valence assigned to $\mathrm{CO}_{2}$ stimuli sensed by BAG neurons. These data indicate that NHR-6 is an essential component of a gene-regulatory mechanism specifically required for the assignment of negative valence to sensory stimuli detected by BAGs. Our data further suggest that the complex physiology of highly specialized neurons such as the BAG neurons of $C$. elegans can be parsed according to genetic programs dedicated to specific physiological characteristics.

\section{Results}

The cell fate determinant ETS-5 binds BAG-specific and pan-neuronal target genes

To identify ETS-5 target genes, we immunoprecipitated ETS-5::GFP encoded by a transgene that rescues the ets5 mutant phenotype (Brandt et al. 2012) and sequenced bound chromatin. ETS-5 is expressed sparsely in the mature nervous system; consistent with a previous study (Brandt et al. 2019), we detected ETS-5::GFP expression in 15 head neurons including BAG (Fig. 1A). We analyzed chromatin sequences enriched by ETS-5 immunoprecipitation using MEME-ChIP (Machanick and Bailey 2011). The most significantly enriched sequence motif proved a near-perfect match to a motif previously determined
A

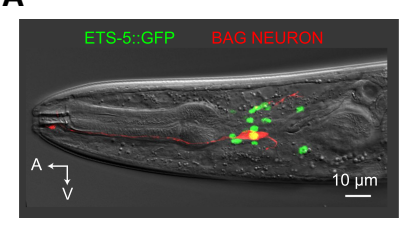

C

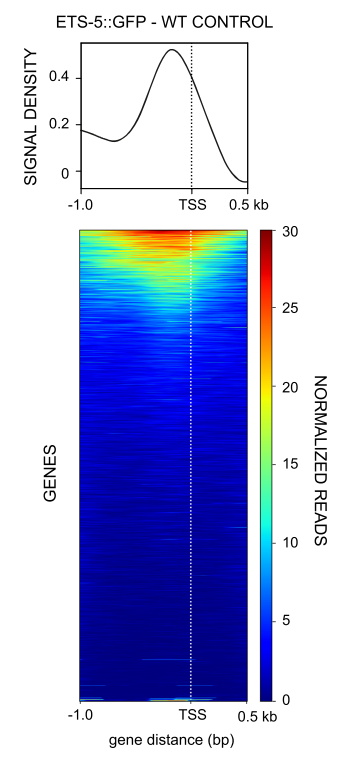

B

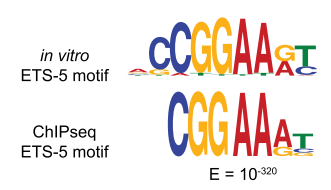

D

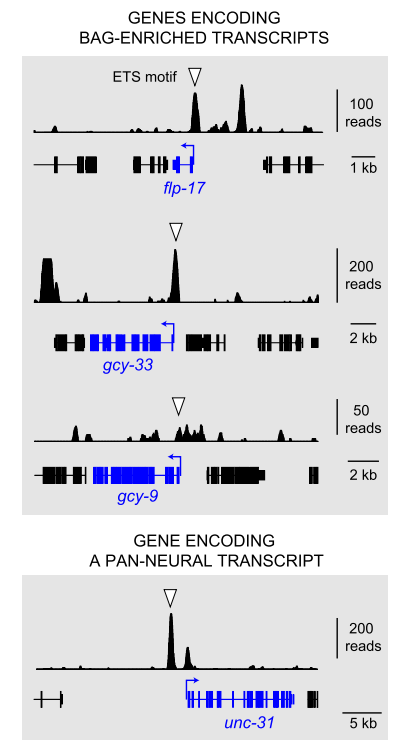

Figure 1. ETS-5 binding is enriched at loci that encode factors specific to BAG chemosensory neurons and at loci that encode pan-neuronal factors. (A) A rescuing ets-5::gfp transgene is expressed in a small number of head neurons, including BAG sensory neurons, which are marked with a Pflp-17::mStrawberry transgene. $(B)$ The most enriched motif in the ETS-5 ChIP-seq data set as determined by MEME-ChIP matches an ETS-5-binding motif determined by a bacterial one-hybrid experiment (Brandt et al. 2019). (C) The distribution of the average ETS-5 ChIP-seq signal near the transcriptional start site (TSS) of genes (top) and per-gene ChIP-seq signals shown as a heat map (bottom). (D) ETS-5 ChIP-seq signals from three loci specifically expressed in BAGs (flp-17, gcy-33, and $g c y-9)$ and a pan-neuronal gene (unc31). Each track shows the mean signal calculated from three independent experiments. 
by in vitro studies of ETS-5 DNA-binding specificity (Fig. 1B; Brandt et al. 2019). From three replicated chromatin immunoprecipitation/sequencing (ChIP-seq) experiments, we identified 1495 binding sites that were detected in each trial. Analysis of the distribution of ETS-5-binding sites within genes revealed that ETS- 5 binding was enriched near transcriptional start sites (Fig. 1C). These data indicate that we successfully purified and sequenced chromatin associated with a sparsely expressed transcription factor that regulates a highly specialized cell fate.

We inspected ETS-5 binding near genes that encode BAG-specific transcripts that we and others have suggested are ETS-5 targets. These genes include the neuropeptide gene $f 1 p-17$ and the guanylyl cyclase genes gcy-33 and gcy-9 (Guillermin et al. 2011; Brandt et al. 2012). Each of these loci contains ETS-5 motifs $5^{\prime}$ to a transcriptional start site (Fig. 1D). We observed large ChIP-seq signals at ETS-5 motifs in the flp-17 locus and the gcy-33 locus. In contrast, ETS-5 binding at the gcy-9 locus was weaker and not restricted to sequences that contained the ETS-5 motif. We also observed evidence for ETS-5 binding sites that did not conform to the motif identified by in vitro and ChIP-seq studies. For example, there was a peak of ETS-5 binding in the $f l p-17$ locus at a site lacking the ETS-5 motif (Fig. 1D). ETS-5 binding was not restricted to loci that encode BAG-specific or BAG-enriched transcripts. Enrichment of ETS-5 binding at an ETS-5 motif in the unc-31 locus, which encodes a pan-neuronal regulator of calcium-dependent neuropeptide secretion, was as pronounced as its binding at sites in loci that encode BAGspecific factors (Fig. 1D). These data suggest that ETS-5 regulates expression of widely expressed and pan-neural genes as well as genes that encode BAG-specific factors. Such a dual role in promoting expression of a specific neuronal fate and in supporting expression of genes required in all neurons has been observed in studies of Pet1, a murine homolog of ets-5, and in studies of other C. elegans transcription factors required for the development of specific circuits (Kratsios et al. 2015; Wyler et al. 2016). These data also made clear that in order to identify ETS-5 target genes with specific functions in BAG neurons we would require a method to measure the effect of ets-5 mutation on transcription in BAG neurons.

\section{An ETS-5-independent and BAG-specific enhancer from the glb-34 locus allows an enumeration of ETS-5-regulated transcripts in BAG neurons}

To measure transcript abundance in ets-5 mutant BAG neurons we sought a BAG-specific reporter transgene that would permit the purification of BAGs by fluorescence-based cell sorting followed by mRNA sequencing. Many BAG-specific reporters exist-for example, reporters based on the neuropeptide gene $f l p-17$, the soluble guanylyl cyclase genes gcy-31 and gcy-33, and the receptortype guanylyl cyclase gene gcy-9-but all require ETS-5 for their expression (Guillermin et al. 2011; Brandt et al. 2012). We therefore sought other genes that might allow us to mark the BAGs of ets-5 mutants by mining the BAG neuron transcriptome for highly enriched transcripts
(Horowitz et al. 2019). We identified glb-34, which encodes a member of a family of globin-like oxygen-binding proteins, as a promising candidate based on enrichment of glb-34 transcripts in BAGs (Fig. 2A). Although glb-34 was not required for BAG-dependent $\mathrm{CO}_{2}$ avoidance behavior (Supplemental Fig. S1), it proved to be the basis of a strong and specific reporter for BAG neurons. A 1-kb fragment upstream of the $g 1 b-34$ transcriptional start site supported BAG-specific expression of GFP in wild-type worms. Expression of this reporter was ETS-5-dependent, as was expression of a reporter using only $223 \mathrm{bp}$ of sequence $5^{\prime}$ to the $g 1 b-34$ transcriptional start (Fig. 2B). We noted the presence of two ETS-5-binding motifs in these reporters (Fig. 2B). Surprisingly, mutation of the ETS-5 motifs in the context of the short reporter construct (each CCGGAA motif was mutated to TTTTTT) did not eliminate reporter expression, and BAG-specific reporter expression persisted in ets-5 mutants (Fig. 2B,C). This short glb-34 reporter, therefore, contains an ETS-5-independent enhancer that is sufficient to support specific expression in BAG neurons. We took the opportunity to use this enhancer to measure the impact of ets-5 mutation on the architecture and function of BAG neurons. Loss of ets5 did not affect the characteristic morphology of BAG neurons, which are bipolar and possess anterior sensory dendrites and a posterior axon that enters the nerve ring to make and receive synaptic connections (Fig. 2C). The sensory dendrites of BAGs possess cilia that are enriched for the cyclic nucleotide-gated channel subunit TAX-4 and the guanylyl cyclase GCY-9 (Martínez-Velázquez and Ringstad 2018). These ciliary markers were properly localized in ets-5 mutants, indicating that ETS-5 is not required for the formation or maintenance of BAG cilia (Fig. 2D). To observe the structure of the BAG axon in more detail, we expressed a fusion of the YFP variant Venus and FLP-17 neuropeptides. This probe marks neurotransmitter release sites (Smith et al. 2013). We observed FLP-17::Venus puncta in the axons of ets-5 mutants, indicating that this probe was properly targeted to release sites in axons. We concluded that ETS-5 is not a critical determinant of the structure of BAG neurons. However, when we used the ratiometric calcium indicator YC3.60 to measure the effect of ets-5 mutation on BAG sensory function we found that ets-5 mutant BAGs were severely compromised for $\mathrm{CO}_{2}$ sensing (Fig. 2E,F). Together, these data show that while ETS-5 is not required for the characteristic structure of BAG neurons, it is critical for their sensory function.

Importantly, the ETS-5-independent enhancer allowed us to mark BAG neurons in ets-5 mutants and purify them for differential gene expression analysis using mRNA-seq. Inspection of known ETS-5 targets ( $f l p-17$, gcy-9, and gcy-33) confirmed that a deletion allele of ets-5 had severe effects on transcription. Inspection of $f l p-17$ transcripts suggested that loss of ETS-5 reduced the number of mRNAs containing exonic sequences and also increased the generation of abnormal transcripts that included intronic sequences and sequences derived from $5^{\prime}$ and $3^{\prime}$ regions of the locus (Fig. 3A). We saw evidence for normal gcy9 and gcy-33 transcripts at greatly reduced levels (Fig. 3A). 
A

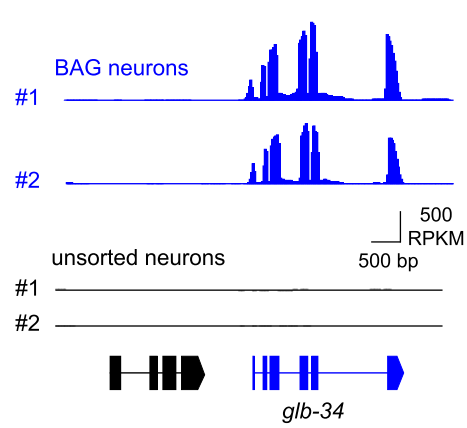

C

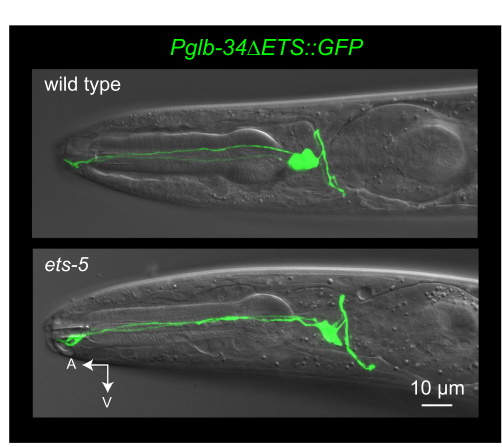

E

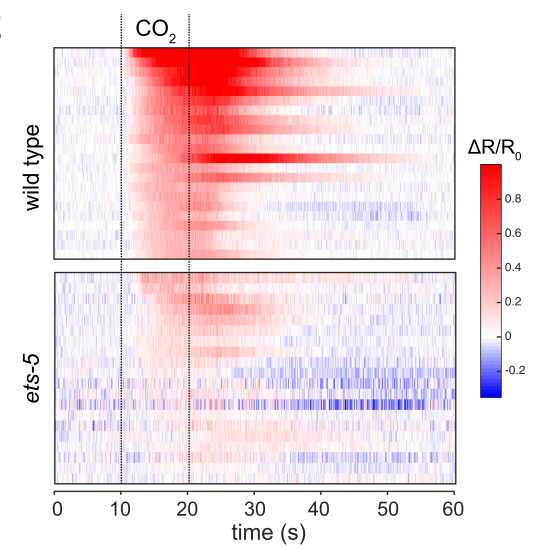

B

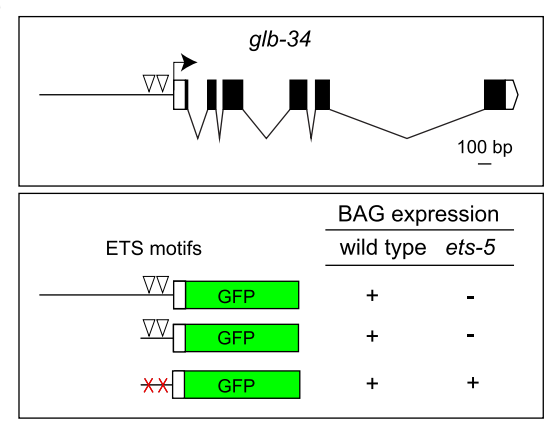

D

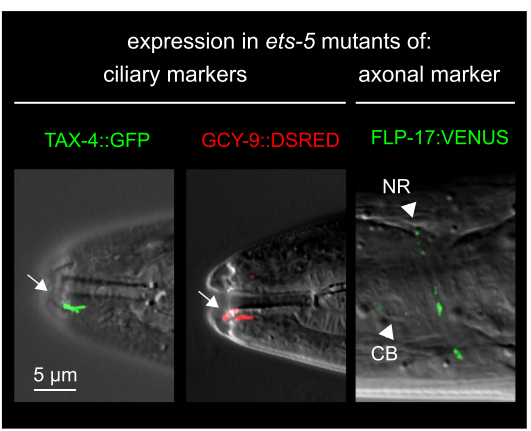

$\mathbf{F}$

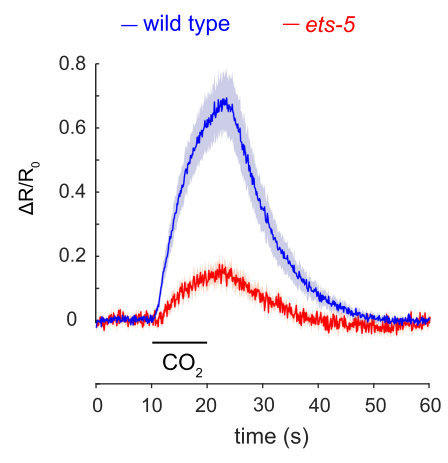

Figure 2. ETS-5 is required for BAG neuron function but is dispensable for BAG neuron architecture. $(A)$ RNA-seq tracks showing enrichment of $g 1 b$-34 transcripts in BAG neurons compared with unsorted cells (Horowitz et al. 2019). (B, top) Gene model of $g l b-34$. (Bottom) Schematics of reporter constructs using long (913-bp) and short (223-bp) cis-regulatory sequences from the $g l b-34$ locus and how expression of these reporters is affected by loss of ETS-5. Open triangles indicate motifs that conform to the ETS-5-binding motif and that were mutated to a poly- $T$ sequence. (C) Micrographs of a wild-type and ets-5 (tm1734) young adult expressing a GFP reporter transgene based on the ETS-5-independent $g l b$-34 enhancer. $(D)$ Reporters for TAX-4 and GCY-9 (cilium) and FLP-17 (axon) expressed in ets-5 mutants under the control of the ETS-5-independent $g l b$ 34 enhancer. (E) Calcium responses of wild-type and ets-5 BAG neurons to $10 \%$ $\mathrm{CO}_{2}$ stimuli measured using the ratiometric calcium indicator YC3.60 and plotted as heat maps to show individual responses. $(F)$ Calcium responses of wild-type and ets5 BAG neurons to $\mathrm{CO}_{2}$ stimuli plotted as mean $\Delta \mathrm{R} / \mathrm{R}_{0}$. Shaded area indicates mean \pm standard error of the mean.
A genome-wide comparison of the transcriptomes of wild-type and ets-5 mutant BAG neurons showed that loss of ETS-5 had different effects on different transcripts. Levels of many transcripts, like gcy-33, gcy-9, and flp-17 transcripts, were reduced in ets-5 BAGs (Fig. 3B). Our data also indicated that expression of $\operatorname{tax}-2$ and $\operatorname{tax}-4$ transcripts, which encode subunits of a cyclic nucleotide-gated ion channel, were unaffected by loss of ETS-5 (Fig. 3B), consistent with a prior study that used transcriptional reporters to determine the effects of ets-5 mutation on gene expression in BAGs (Guillermin et al. 2011).

Down-regulated and up-regulated transcripts were assigned different functional classes by gene ontology analysis (Fig. 3C,D). Many transcripts that were downregulated in ets-5 BAGs encode transcription factors (Fig. 3C). We therefore inspected more closely loci that encode transcription factors known to be critical for BAG function. Expression of a short isoform of VAB-3, a C. elegans Pax6 homolog that is required for proper
BAG development (Brandt et al. 2019), was reduced in ets-5 BAGs (Fig. 3E). So too were egl-13 transcripts (Supplemental Fig. S2), which encode a Sox domain-containing transcription factor required for development of BAGs (Gramstrup Petersen et al. 2013). Levels of a BAG-enriched transcript encoding an uncharacterized homeodomain-containing transcription factor, CEH-54, were also strongly reduced by loss of ETS-5 (Fig. 3E). We generated mutants lacking ceh-54 to determine whether it is required for BAG function and found that ceh-54 mutants were only weakly defective for BAG-dependent $\mathrm{CO}_{2}$ avoidance and that we could not rule out the possibility that this difference was caused by experimental error (Supplemental Fig. S3). Together, these data indicate that the set of transcripts down-regulated by loss of ETS-5 contains transcription factors that are either known to be important for BAG function or whose sparse expression pattern implicates them in BAG neuron development. 
A
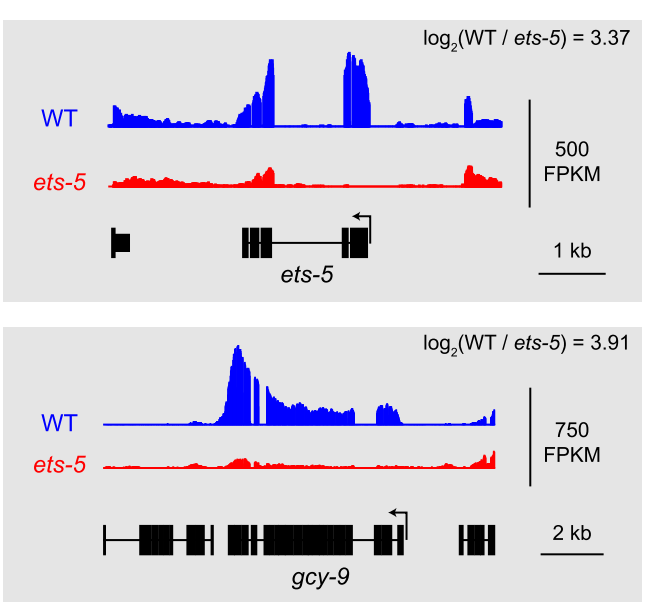

B

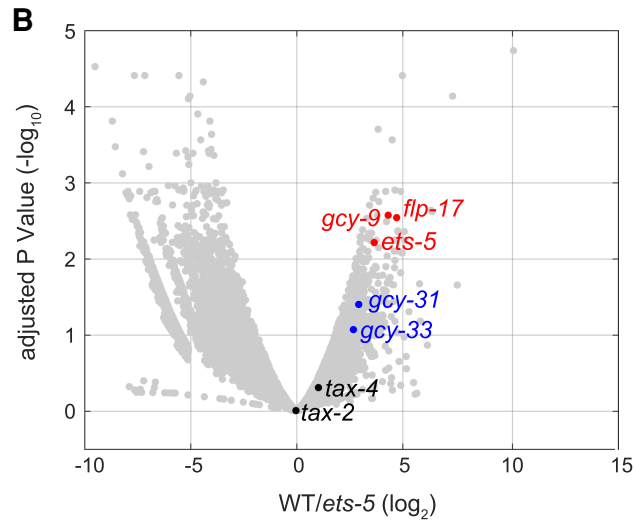

D

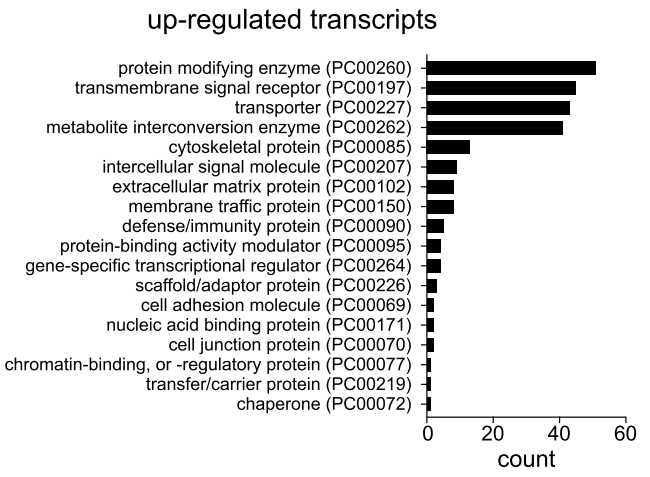

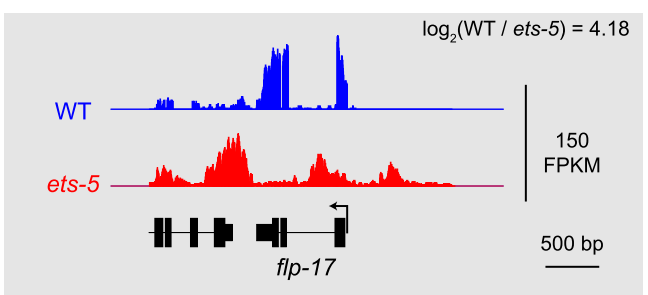

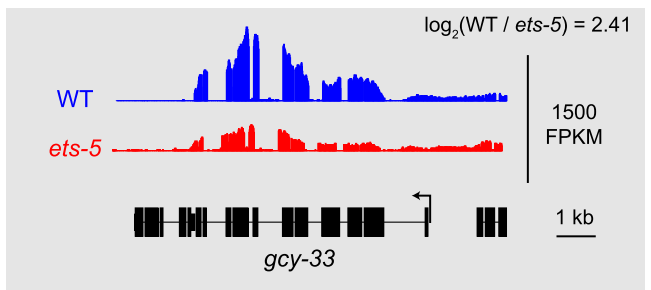

C

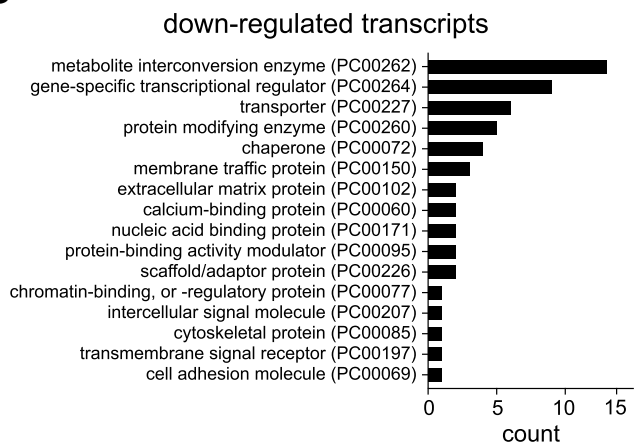

$\mathbf{E}$

BAG-enriched transcription factors

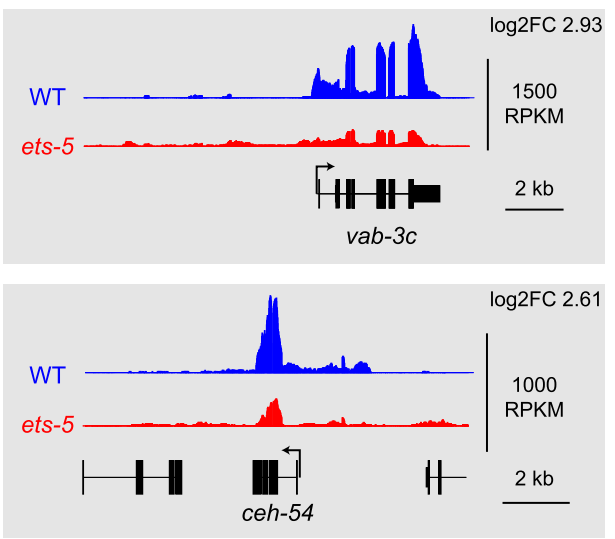

Figure 3. ets-5 mutation disrupts expression of BAG-specific genes and causes widespread changes to the BAG neuron transcriptome. (A) RNA-seq tracks from wild-type and ets-5 BAG neurons showing the effect of ets-5 mutation on BAG-enriched transcripts. Data are plotted as fragments per kilobase of transcript per million mapped reads (FPKM). $\log _{2}$ fold changes in transcript levels are indicated in the top right corner of each panel. $(B)$ Volcano plot showing the per-gene effect of ets-5 mutation on gene expression in BAG neurons. Some canonical markers of a BAG fate are indicated by colored data points. $(C)$ Gene ontology terms associated with genes down-regulated in ets-5 BAG neurons identified by PANTHER. (D) Gene ontology terms associated with genes up-regulated in ets-5 BAG neurons identified by PANTHER. (E) RNA-seq tracks for loci encoding the BAG-enriched homeodomain-containing factors $v a b-3 c$ and $c e h-54$.

Integrating ChIP-seq and RNA-seq data identifies an NR4A family nuclear receptor required for BAG neuron function

We determined intersections of sets of genes identified by ETS-5 ChIP-seq, genes down-regulated or up-regulated in ets-5 mutant BAG neurons, and genes that encode BAG-enriched transcripts. Nearly one-fifth of genes that were down-regulated in ets-5 mutant BAGs encoded BAG-enriched transcripts (Fig. 4A). In contrast, less than one percent of up-regulated genes encoded BAG-enriched transcripts (Fig. 4A). We also noted that down-regulated 
A

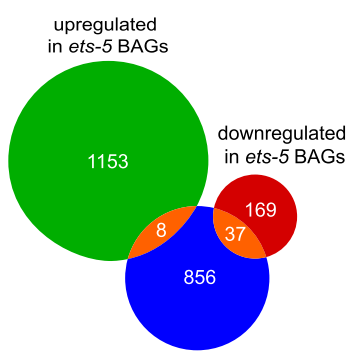

BAG-enriched transcripts

C

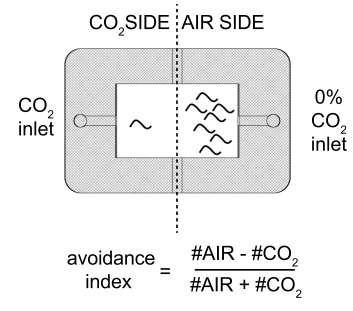

B

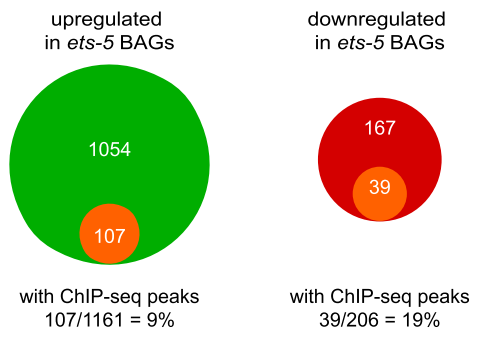

D

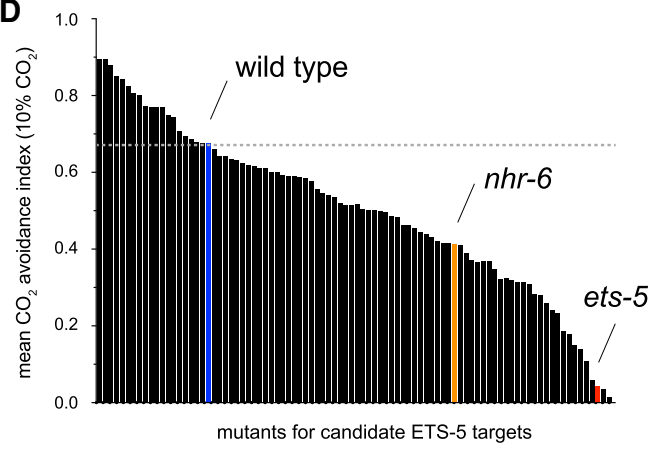

Figure 4. A screen of ETS-5 target genes identifies factors required for BAG chemosensory function. $(A)$ Venn diagram showing overlap between BAG-enriched and ets-5-regulated genes defined as genes whose transcripts displayed a $\log _{2}$ fold change $>2$ in ets-5 mutant BAGs. $(B)$ Venn diagrams illustrating the fraction of ETS5- up-regulated and down-regulated genes having ETS-5 ChIP-seq peaks within $5 \mathrm{~kb}$ of their coding sequence. $(C)$ Schematic of the $\mathrm{CO}_{2}$ avoidance assay used to measure BAG function. (D) Bar graph displaying the mean $\mathrm{CO}_{2}$ avoidance index of mutants for ETS-5-regulated genes. Avoidance of each strain to $10 \% \mathrm{CO}_{2}$ was measured at least three times. genes are more than twice as likely to be within $5 \mathrm{~kb}$ of an ETS-5 ChIP-seq peak than are up-regulated genes (Fig. 4B). These data supported our hypothesis that ETS-5 directly promotes expression of factors specific to BAG, and we therefore executed a screen for functionally important ETS-5 target genes with a focus on genes at the intersection of our ChIP-seq and RNA-seq data sets.

We assembled a panel of 84 mutants carrying null alleles of presumptive ETS-5 target genes. To determine whether a candidate gene was required for BAG neuron function, we subjected the corresponding mutant to a $\mathrm{CO}_{2}$ avoidance assay in which animals were placed in an arena containing a high $(10 \%) \mathrm{CO}_{2}$ zone and a control zone with no $\mathrm{CO}_{2}$ (Fig. 4C). After $35 \mathrm{~min}$, the number of animals in each zone was counted and $\mathrm{CO}_{2}$ avoidance index was computed as shown in Figure 4C. Candidate genes included some with known roles in chemosensation; for example, odr-2 (Chou et al. 2001) and pdl-1 (Fig. 4D; Supplemental Table S1; Gross et al. 2014). Our initial screen also identified candidates that did not withstand further scrutiny. Strains containing null alleles of $r g s-6$, which encodes a regulator of GPCR signaling, and $R 11 G 1.1$, which encodes an uncharacterized LDL receptor-like protein, were defective for $\mathrm{CO}_{2}$ avoidance. However, independently generated deletion alleles of these genes displayed weaker defects in avoidance behavior (Supplemental Fig. S4), suggesting that other unknown mutations might contribute to the avoidance defects we observed in our initial screen.

Our functional screen of ETS-5 targets also identified nhr-6 (Fig. 4D), which encodes the sole C. elegans member of the NR4A family of nuclear receptors (Supplemental Fig. S5). According to our $\mathrm{ChIP}=$ seq and mRNA-seq analyses, there was a prominent peak of ETS-5 binding at an ETS- 5 motif in the $n h r-6$ locus and loss of ETS- 5 greatly re- duced nhr-6 transcript levels in BAGs (Fig. 5A). A fosmidbased $n h r-6:: g f p$ reporter transgene revealed that $n h r-6$ was expressed in the nuclei of a small number of head neurons, including the BAGs (Fig. 5B). This is consistent with the first studies of $n h r-6$ expression, which found that it was expressed prominently in the spermatheca of young hermaphrodites but also in a small number of head neurons (Gissendanner et al. 2008). We compared expression of NHR-6::GFP in the wild type with its expression in ets-5 mutants and found that loss of ETS-5 reduced expression of NHR-6::GFP in BAGs (Fig. 5B,C), consistent with the effect we observed of ets-5 mutation on nhr-6 transcript levels in BAGs. Unlike ets-5, which is expressed shortly after the BAG neuron is born (Brandt et al. 2019), $n h r-6$ expression arises late in development and is only detected at early larval stages or just before hatching (Supplemental Fig. S6). We tested whether other regulators of BAG fate were required for expression of $n h r-6$ in BAGs, and we found that $n h r-6$ expression also required the p38 MAP kinase PMK-3 and the transcription factor EGL-13 (Supplemental Fig. S7). These data indicated that $n h r-6$ is expressed in BAGs, where its expression is coordinately regulated by ETS- 5 and other factors that promote the BAG neuron fate.

\section{NHR-6 is required for $B A G$ neuron function and regulates gene expression in $B A G$ neurons}

To test whether nhr-6 expression in BAG neurons is required for BAG neuron function, we used a combination of transgene rescue experiments and cell targeted RNAi to manipulate $n h r-6$ function and determine the effect on $\mathrm{CO}_{2}$ avoidance. nhr-6 mutants were showed an intermediate defect in avoidance of $10 \% \mathrm{CO}_{2}$ stimuli (Fig. 4D), which are strong stimuli that saturate the BAG 
A

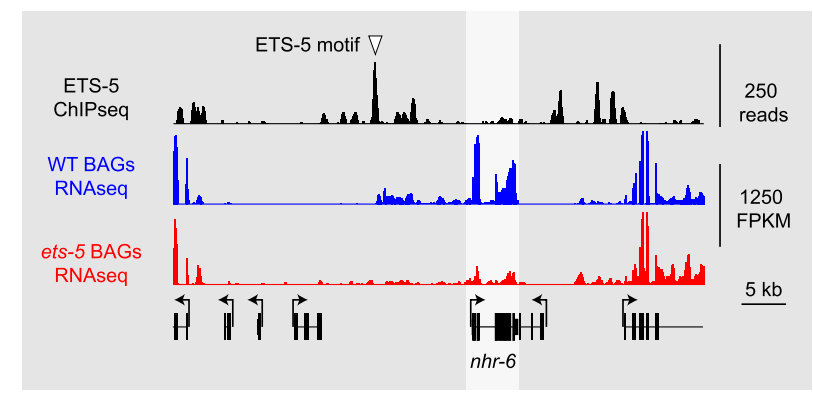

B

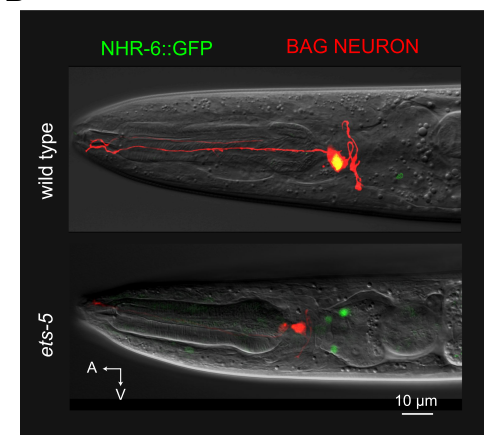

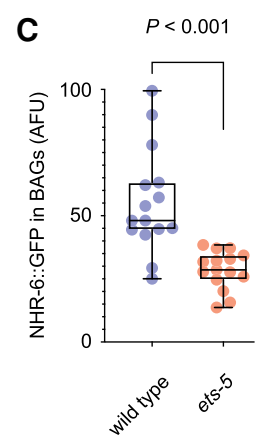

Figure 5. The Nr4A-type nuclear receptor gene $n h r-6$ is an ETS5 target whose expression is highly enriched in BAG neurons. (A) ETS-5 ChIP-seq and RNA-seq tracks for the nhr-6 locus showing the presence of an ETS-5-binding site coincident with an ETS5-binding motif (open triangle) and the effect of ets-5 mutation on transcript abundance. $(B)$ Micrographs of a wild-type and ets-5 (tm1734) young adult coexpressing NHR-6::GFP and the red fluorescent BAG marker Pflp-17::mStrawberry. (C) Scatter plot of quantified NHR-6::GFP fluorescence intensities in the wildtype and ets-5 mutants. (AFU) Arbitrary fluorescence units.

chemotransduction machinery (Hallem et al. 2011b). We found that $2 \% \mathrm{CO}_{2}$ stimuli, which elicit from the wild type a robust avoidance response, are not avoided by nhr-6 mutants (Fig. 6A), indicating that the sensitivity of the avoidance response was severely blunted by loss of nhr-6. The fosmid-based nhr-6::gfp transgene that we used to determine the expression of nhr- 6 in the nervous system restored $\mathrm{CO}_{2}$ avoidance to nhr-6 mutants. In a complementary set of experiments, we used transgenegenerated RNAi to knock down nhr-6 function either in all neurons or only in BAG neurons. Pan-neuronal RNAi caused a defect in $\mathrm{CO}_{2}$ avoidance, as did specific knockdown of $n h r-6$ in BAG neurons. We noted that the nhr-6 locus is predicted to encode two NHR-6 isoforms with different transcriptional start sites. Available nhr-6 alleles delete sequences common to both isoforms (Supplemental Fig. S8). We generated an allele of nhr-6 that deletes sequences unique to the long NHR-6 isoform, and we found that mutants carrying this allele were normal for $\mathrm{CO}_{2}$ avoidance (Supplemental Fig. S8). These data indicate that a short NHR-6 variant containing ligand-binding and DNA-binding domains suffices to support BAG function and that a longer NHR-6 isoform with an extended amino terminus is dispensable. Importantly, these data also show that the NHR-6 nuclear receptor functions cell-autonomously in BAGs to promote $\mathrm{CO}_{2}$ avoidance behavior.

Because nuclear receptors regulate transcription, we asked whether $n h r-6$ mutants were defective for expression of genetic markers of a BAG neuron fate. Expression of the neuropeptide gene $f l p-17$ or the receptor-type guanylyl cyclase gene gcy-9 was not affected by loss of nhr6 (Fig. 6B). In contrast, loss of nhr-6 significantly reduced expression of the soluble cyclase genes gcy-31 and gcy33 (Fig. 6B). These data indicated that NHR-6 regulates expression of a subset of genes that are regulated by ETS- 5 .

\section{NHR-6 is required to assign negative valence to $\mathrm{CO}_{2}$ stimuli but not for BAG sensory function}

We considered two distinct models for NHR-6 function. First, we considered the possibility that NHR-6 is required for $\mathrm{CO}_{2}$ sensing by BAG neurons; e.g., its function is similar to that of ETS-5 (Fig. 7A). Unlike ETS-5, however, NHR-6 was not required for expression of $g c y-9$, which encodes a receptor-type guanylyl cyclase that likely acts as a receptor for $\mathrm{CO}_{2}$ (Smith et al. 2013) and is necessary for physiological responses of BAG neurons to $\mathrm{CO}_{2}$ stimuli (Guillermin et al. 2011). We tested whether NHR-6 might regulate expression of other factors required for chemosensitivity by measuring $\mathrm{CO}_{2}$-evoked calcium responses of BAG neurons in the wild type and in nhr-6 mutants. Unlike ets-5 mutant BAGs, which displayed severely blunted calcium responses to $\mathrm{CO}_{2}$ stimuli (Fig. 2E-F), BAG neurons of $n h r-6$ mutants responded robustly to $\mathrm{CO}_{2}$ stimuli (Fig. 7B). A quantification of the magnitudes and dynamics of the responses nhr- 6 mutant BAGs revealed no significant effect of $n h r-6$ mutation on the size of the calcium response and only a small decrease in the time of recovery to baseline calcium levels after the offset of $\mathrm{CO}_{2}$ stimuli (Supplemental Fig. S9). Therefore, unlike ETS-5, NHR-6 does not seem to be required for the expression of factors that transduce $\mathrm{CO}_{2}$ stimuli into neural activity. These data did not support a model in which NHR6 promotes chemosensitivity of BAG neurons, as does ETS-5, and prompted us to consider an alternate model. Recent studies show that in response to developmental or experiential cues, BAG neurons switch from supporting $\mathrm{CO}_{2}$ avoidance to driving attraction to $\mathrm{CO}_{2}$ stimuli (Hallem et al. 2011a; Guillermin et al. 2017; Bhattacharya et al. 2019; Rengarajan et al. 2019). This plasticity is a critical aspect of BAG neuron function because the $\mathrm{CO}_{2}$ that they sense is a proxy for the abundance and quality of environmental microbes, on which C. elegans feeds. By switching the valence of $\mathrm{CO}_{2}$ stimuli, BAG neurons are able to generate food-response behaviors that adaptively meet the animal's metabolic requirements.

We next tested the possibility that NHR-6 is required for assigning valence to $\mathrm{CO}_{2}$ stimuli (Fig. 7A). We subjected animals to food-deprivation protocols that cause the wild type to switch from $\mathrm{CO}_{2}$ avoidance to $\mathrm{CO}_{2}$ attraction (Rengarajan et al. 2019) and determined how $\mathrm{CO}_{2}$ response behaviors of nhr- 6 mutants were affected. As expected, the wild type switched from avoidance to 
A

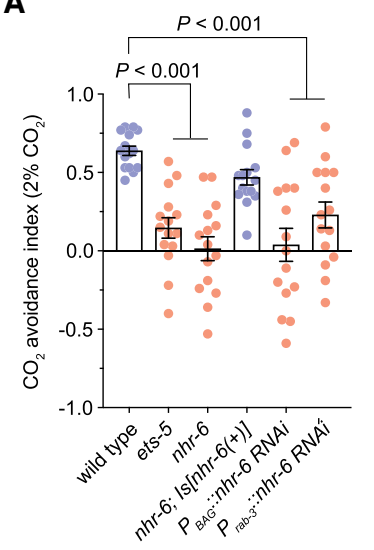

B

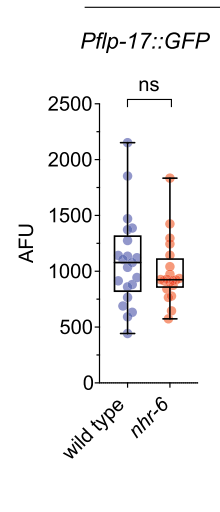

BAG neuron fate markers

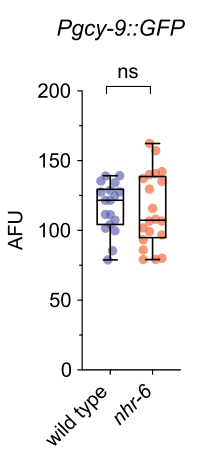

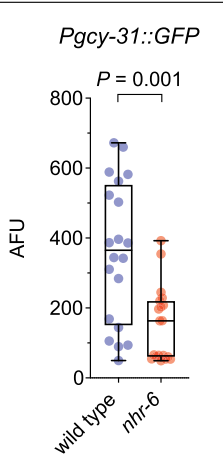

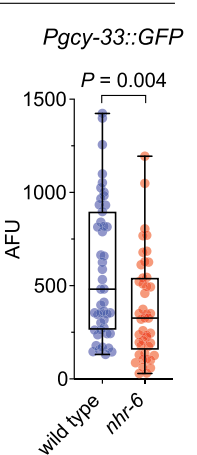

Figure 6. $n h r-6$ is required for $\mathrm{CO}_{2}$ avoidance and regulates expression of a subset of BAG-specific genes. $(A)$ Avoidance indices to $2 \%$ $\mathrm{CO}_{2}$ of strains carrying a deletion allele of $n h r-6$, an $n h r-6$ mutant carrying a rescuing NHR- $6:$ GFP transgene, and transgenes generating BAG-specific and pan-neural knockdown of $n h r-6$ using flp-17 and rab-3 cis-regulatory sequences, respectively. (B) nhr- 6 mutants exhibit reduced expression of $g c y-31$ and $g c y-33$ reporter transgenes but have normal expression of $g c y-9$ and $f l p-17$ reporter transgenes.

attraction after either 2.5 or $5 \mathrm{~h}$ of fasting (Fig. $7 \mathrm{C}$ ). ets-5 mutants, which fail to sense $\mathrm{CO}_{2}$ stimuli, were defective for attraction to $\mathrm{CO}_{2}$ stimuli after fasting just as they were defective for avoidance of $\mathrm{CO}_{2}$ under basal conditions. In contrast, $n h r-6$ mutants, which were profoundly defective for $\mathrm{CO}_{2}$ avoidance under basal conditions, were fully competent for chemotaxis toward $\mathrm{CO}_{2}$ stimuli after fasting (Fig. 7C). We saw the same using BAG cell targeted knockdown of $n h r-6$. Whereas selective loss of $n h r-6$ in BAG neurons caused a $\mathrm{CO}_{2}$ avoidance defect (Fig. 6A), it had no measurable effect on BAG-mediated $\mathrm{CO}_{2}$ attraction (Supplemental Fig. S10). Because many nuclear receptors are regulated by mechanisms that shuttle them in and out of the nucleus (Evans and Mangelsdorf 2014), we tested whether feeding state regulated the nuclear localization of NHR-6. We found, however, that the nuclear localization of NHR-6 did not measurably change upon fasting (Supplemental Fig. S10), suggesting that if NHR6 is regulated by feeding state then it is regulated by mechanisms other than nuclear translocation. These data indicate that NHR-6 is not essential for the general chemosensory function of BAG neurons but is specifically required for BAGs to assign a negative valence to $\mathrm{CO}_{2}$ stimuli.

\section{Discussion}

Developmental programs endow the chemosensory BAG neurons with mechanisms that support their chemosensory function and also with a plasticity mechanism that switches BAGs from mediators of avoidance to mediators of attraction. Our study suggests that the generation of such a specialized neuron type is accomplished by a hierarchy of transcriptional regulators. Shortly after they are generated, embryonic BAG neurons express the ETS family transcription factor ETS-5 (Brandt et al. 2019). In addition to directly regulating genes required for chemosensation, intracellular signaling, and neurotransmission, we found that ETS-5 regulates expression of a battery of transcription factors, many of which are required for BAG function. These include the homeodomain-containing factors VAB-3 and CEH-54, the Sox domain-containing factor EGL-13, and the Nr4a-type nuclear receptor NHR-6.

There is similarity between the role of ETS-5 in BAG neuron development and the function of its vertebrate homolog Pet1, which is required for the development of hindbrain serotonergic neurons. Some Pet1 neurons are, like BAG neurons, endowed with $\mathrm{CO}_{2}$ chemosensitivity, which allows them to monitor levels of blood gases and control breathing (Erickson et al. 2007; Ray et al. 2013). The importance of the chemosensory function of PET1 neurons in respiratory physiology has been demonstrated both under basal conditions and under conditions of respiratory distress (Ray et al. 2011; Dosumu-Johnson et al. 2018). PET1 directly promotes expression of genes required for a serotonergic fate; e.g., genes required for serotonin biosynthesis and transport. PET1 also promotes expression of a set of transcriptional regulators, including the homeodomain-containing factors Engrailed1 and Proxl, the Sox domain-containing factors Sox 9 and Sox14, and the NR1 family nuclear receptor RorB (Wyler et al. 2016). How PET1 neurons in the hindbrain sense blood gases remains unknown. Based on our study of ETS-5, we suggest that the mechanisms of $\mathrm{CO}_{2}$ chemosensitivity in PET1 neurons might be encoded by genes directly regulated by PET1 or regulated by transcription factors induced by PET1 during development.

It is striking that despite the critical roles for ETS- 5 and PET1 in development of specialized neuron types, in the absence of these factors, neurons are generated that still have many of the features of their wild-type counterparts. For example, the BAG neurons of ETS-5 mutants display the characteristic morphology of a normal BAG neuron. Although ETS-5 is required for expression of the machinery for chemosensation, it is not required for the generation of a sensory cilium that, to a first approximation, 
A

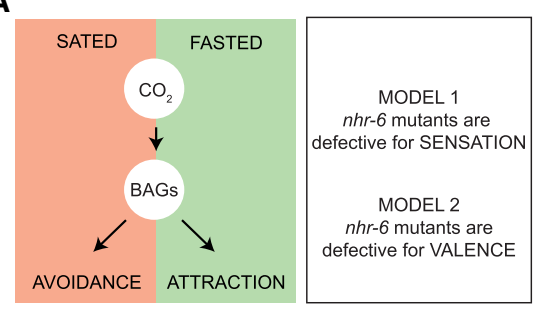

B
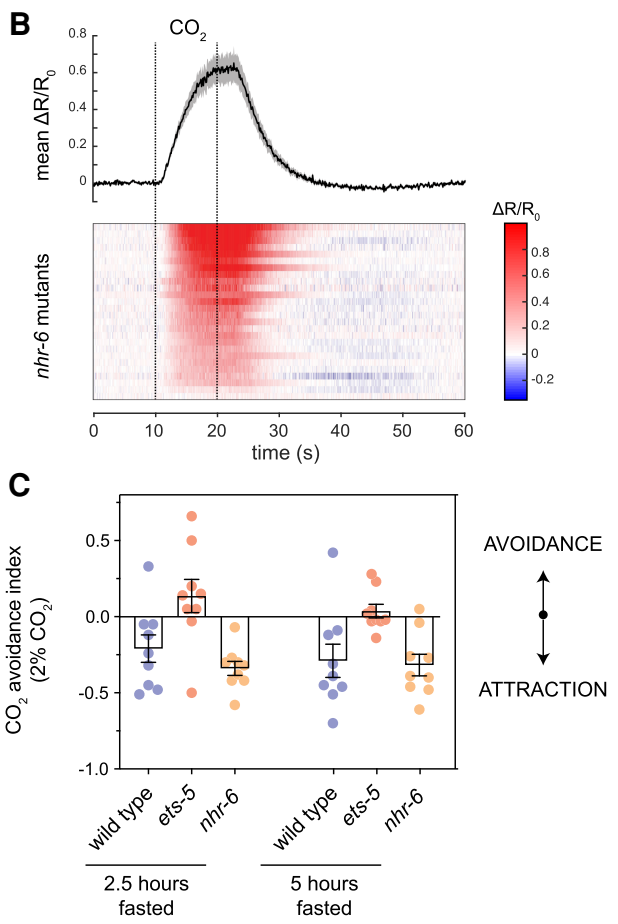

Figure 7. $n h r-6$ is required for the assignment of negative valence to $\mathrm{CO}_{2}$ stimuli. (A) A schematic of BAG neuron plasticity showing alternate models of NHR-6 function in BAG. $(B)$ Calcium responses of $n h r-6$ mutant BAG neurons using the ratiometric calcium indicator YC3.60. The solid line is mean $\Delta R / R_{0}$ and the shaded area represents \pm standard error of the mean. A heat map of individual calcium responses is shown in the bottom panel. $(C)$ Avoidance indices to $2 \% \mathrm{CO}_{2}$ stimuli of the wild-type, ets-5 mutants, which are $\mathrm{CO}_{2}$-blind, and $n h r-6$ mutants under basal conditions and after 2.5 or $5 \mathrm{~h}$ of fasting.

has normal morphology. Without ETS-5, BAG neurons do not express neuropeptides critical for chemotaxis, but reexpression of tagged neuropeptides in ets-5mutants revealed that BAG neuron axons are properly differentiated and accumulate neuropeptides in puncta that are similar to those seen in wild-type neurons (Smith et al. 2013). These data indicate that the cellular architecture of BAG neurons is specified by an ETS-5-independent mechanism. Because ETS-5 is required for expression of factors that require specific cellular compartments for their function (GCY-9 requires the cilium to function in chemosensation and FLP-17 neuropeptides require a neurite differentiated into an axon), it is likely that factors that promote BAG neuron morphogenesis and the generation of specialized cellular compartments precede ETS-5 during development. It will be interesting to determine how morphogenic mechanisms, whose molecular constituents are unknown, are coordinated with ETS-5-dependent mechanisms to generate a fully differentiated BAG neuron.

Nuclear receptors have conserved roles in patterning and refining neuronal cell fates during nervous system development

We focused our study on one of the transcriptional regulators induced by ETS-5 during BAG development: the nuclear receptor NHR-6. The C. elegans genome encodes an extraordinary number (284) of nuclear receptors, most of which are members of nematode-specific outgroups and whose functions are unknown (Taubert et al. 2011). NHR-6, however, is not part of the expanded nematode-specific clade of nuclear receptors. It is conserved and homologous to three vertebrate proteins that constitute the Nr4a family of nuclear receptors: NR4A1/ Nurr77, NR4A2/Nurr1, and NR4A3/Nor1. NHR-6 has a well understood role outside of the nervous system in the developing spermatheca, where it promotes cell cycle progression (Gissendanner et al. 2008, 2014; Heard et al. 2010; Praslicka and Gissendanner 2015). In BAG neurons, however, nhr- 6 is expressed after a BAG fate has been established and long after the BAG last cell division in the lineage that creates BAGs, indicating that its role in sensory neuron development is distinct from its role in the development of nonneural tissues.

There are several examples of NHR-6-like factors regulating nervous system development. NR4A nuclear receptors are expressed in the developing vertebrate nervous system and, like NHR-6, are required for the function of specific neuron types. A striking example of the requirement for an NR4A nuclear receptor in nervous system development is NR4A2/Nurr1. This nuclear receptor is expressed in midbrain dopamine neurons, where it is required for a dopaminergic fate and for cell survival (Zetterström et al. 1997; Zhang et al. 2012). Just as NHR-6 expression is regulated by ETS-5, a cell fate determinant expressed in the newly born BAG neuron, NR4A2/ Nurrl expression is promoted by Lmxla, whose expression is activated in immature dopamine neurons of the vertebrate midbrain (Hoekstra et al. 2013). The phenotype associated with mutation of another vertebrate NHR-6 homolog, NR4A3/Nor1, suggests that it also functions to regulate gene expression in specific neural circuits and might function in brain development. NR4A3/Nor1 mutates to cause seizures and is specifically required for cell survival and proper circuit assembly in the hippocampus (Pönniö and Conneely 2004). Our data suggest that NR4A-type nuclear receptors have an evolutionarily ancient role in nervous system development, specifically in regulating expression of cellular characteristics that define functionally specialized neuron types.

It is interesting to compare the function of NR4A-type nuclear receptors with those of COUP-TF/Nr2b nuclear receptors and RAR/RXR retinoic acid receptors, which also play critical roles in nervous system development. 
Unlike C. elegans NHR-6 and mammalian Nurr1, which function in postmitotic neurons to promote expression of specific neuronal fates, COUP-TF1 and RAR/RXR nuclear receptors have earlier roles in establishing domains within the embryonic nervous system that will ultimately generate a specific set of neuron types. COUP-TF1 is expressed in neural precursors in the forebrain, where it regulates the number of neurons in developing cortex. RAR/RXR nuclear receptors also act early in nervous system development as morphogen receptors that establish domains of expression of cell fate determinants in the developing hindbrain and spinal cord (Wilson et al. 2004; Glover et al. 2006). An early role for a nuclear receptor in nervous system development does not preclude a later role. RAR/RXR retinoid receptors are required continuously for neuron survival and for maintenance of certain neuronal fates. Also, COUP-TF1 expression continues after neurogenesis in the developing forebrain, where it promotes cortical regionalization (Hu et al. 2017). The $C$. elegans COUP-TF1 homolog UNC-55 similarly acts in postmitotic motor neurons to generate classes of motor neuron that integrate into distinct circuits for forward and reverse locomotion (Walthall and Plunkett 1995; Zhou and Walthall 1998; Petersen et al. 2011; He et al. 2015; Miller-Fleming et al. 2016). Our study indicates a late role for NHR-6 in the development of the C. elegans nervous system analogous to those of NR4A2/Nurr1 and UNC-55. Like these nuclear receptors, NHR-6 promotes further differentiation and specialization of a neuron type after it has been born and after it has acquired some of its characteristics. In fact, we did not even observe expression of NHR- 6 in the nervous system until the very end of embryonic development, long after BAG neurons were born and after they display hallmarks of differentiation; e.g., expression of BAG-specific factors.

\section{A developmental module required for neural plasticity}

Previous work demonstrated that the response of C. elegans to $\mathrm{CO}_{2}$ stimuli is highly context-dependent. $\mathrm{CO}_{2}$ stimuli can be aversive or attractive depending on prior $\mathrm{CO}_{2}$ exposure and feeding state (Guillermin et al. 2017; Rengarajan et al. 2019). Certain BAG neuropeptides have been implicated in promoting either attraction or avoidance (Guillermin et al. 2017); however, the nhr-6 mutant phenotype is unique in exhibiting defective avoidance while being competent for attraction. The closest phenotype to that caused by mutation of $n h r-6$ is the phenotype of animals that lack dopamine neurons, which are also defective for $\mathrm{CO}_{2}$ avoidance and competent for $\mathrm{CO}_{2}$ attraction. These mutants, however, display constitutive attraction to $\mathrm{CO}_{2}$ regardless of feeding state and are very likely defective in modulation of the chemotaxis circuit downstream from BAGs (Rengarajan et al. 2019). In contrast, NHR-6 is required in BAG neurons to support $\mathrm{CO}_{2}$ avoidance, demonstrating the existence of a BAG cell-intrinsic mechanism required for associating valence with sensory stimuli.

The genetics of BAG neuron development suggest an underlying modular architecture of the mechanisms that generate these highly specialized sensory neurons. ETS-5 and NHR-6 are not required for the generation of a sensory neuron-like cell with bipolar morphology and a sensory cilium containing effectors of chemotransduction such as TAX-2 and TAX-4 channels. Without ETS-5, BAG neurons do not function as gas sensors, suggesting that ETS-5 is an integral part of a module that confers a specific chemosensory function-sensing the respiratory gas $\mathrm{CO}_{2}-$ to neurons already programmed to be chemosensors. $n h r-6$, as an ETS-5 target gene, is a part of this module that is not required for BAG chemosensory function per se but is required for assigning valence to $\mathrm{CO}_{2}$ stimuli according to feeding state. We suggest that NHR-6 and its gene targets constitute a developmental module that adds to specialized chemosensory neurons the ability to switch valence, which supports functional plasticity. NHR-6 might be required for the switching process, in which case the defective avoidance behavior of $n h r-6$ mutants would be caused by incomplete acquisition of a state that assigns negative valence to $\mathrm{CO}_{2}$ stimuli. It is also possible that NHR-6 is required for expression of factors necessary for negative valence that act downstream from valence switching. In this case, BAG neurons are incapable of generating the signal that assigns negative valence to chemosensory stimuli but remain able to generate the signals corresponding to positive valence. These alternate models can be tested once we understand the cell-intrinsic physiological and molecular mechanisms that allow BAG neurons to switch valence.

Our study indicates that there is a process intrinsic to BAGs that mediates valence switching and that this process requires the NHR- 6 transcriptional regulator. It is possible that expression of NHR-6 is sufficient to endow neurons with the ability to similarly modulate their functions in response to feeding state or metabolic cues. One way this might happen is via regulation of NHR-6 by a metabolite that serves as a ligand. Mammalian NR4A family transcription factors bind to and are regulated by a myriad of small molecules, including some that resemble products of lipid metabolism (Zhan et al. 2008). This raises the intriguing possibility that NHR-6, perhaps along with its vertebrate homologs, is normally regulated by metabolic signals, and that this regulation is part of its role in the plasticity of BAG neurons. Whether or not NHR-6 function can be transferred to other neuron types, our study predicts that some NHR-6 target genes will, like $n h r-6$ itself, be required specifically for $\mathrm{CO}_{2}$ avoidance and therefore part of the mechanism that confers functional plasticity to BAG neurons. Discovery of such NHR-6 targets and how their roles in BAG neuron physiology will allow us to determine the molecular mechanisms that endow BAGs with the remarkable ability to flexibly assign valence to sensory stimuli.

\section{Materials and methods}

Strains used in this study

Strains used in this study in the order in which they appear were as follows: FQ237 wzIs80[ETS-5::gfp lin-15(+)]; lin-15AB(n765) X, 
FQ1814 wzIs135[Pglb-344ETS-5::gfp] I, FQ1854 wzIs135[Pglb34DETS-5::gfp] I; ets-5(tm1734) X, FQ1428 wzEx400[Pglb-34(1):: gfp; Punc-122::mCherry], FQ1446 ets-5(tm1734) X; wzEx400 [Pglb-34(1)::gfp; Punc-122::mCherry], FQ1482 wzEx409[Pglb-34 (s)::gfp; Punc-122::mCherry], FQ1535 ets-5(tm1734) X; wzEx409 [Pglb-34(s)::gfp; Punc-122::mCherry], FQ2304 ets-5(tm1734) X; wzEx561[Ppes-10::2xglb-34LETS-5::tax-4::gfp; Punc-122::gfp], FQ2370 ets-5(tm1734) X; wEx567[Ppes-10::2xglb-34LETS-5::gcy9::gfp; Punc-122::gfp], FQ2356 ets-5(tm1734) X; wEx585[Ppes-

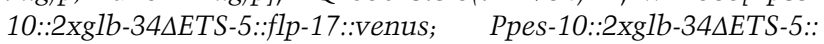
rab-3::mCherry; Punc-122::gfp], FQ2450 nhr-6(lg6001) III; wzEx535[Ppes-10::2xglb-34AETS-5::YC3.60; Punc-122::gfp], FQ2041 ets-5(tm1734) X; wzEx535[Ppes-10::2xglb-34AETS-5:: YC3.60; Punc-122::gfp], FQ2032 wzEx535[Ppes-10::2xglb34AETS-5::YC3.60; Punc-122::gfp], FX01734 ets-5(tm1734) X, DN20 nhr-6(lg6001) III, FX00870 nhr-6(tm870) III, OP90 unc-119 (ed3) III; wgIs90[nhr-6::TY1::EGFP::3xFLAG + unc-119(+)], FQ2425 nhr-6(1g6001) III; wgIs90[nhr-6::TY1::EGFP::3xFLAG + unc-119(+)], FQ2506 unc-119(ed3) III; ets-5(tm1734) X; wgIs90 [nhr-6::TY1::EGFP::3xFLAG + unc-119(+)]; WzEx615[Ppes10::2xglb-34LETS-5::dsRed], FQ2518 unc-119(ed3) III; egl-13 (ku194) X; wgIs90[nhr-6::TY1::EGFP::3xFLAG + unc-119(+)], FQ2526 unc-119(ed3) III; wgIs90[nhr-6::TY1::EGFP::3xFLAG + unc-119(+)]; wzEx36[Pflp-17::dsRed], FQ2564 unc-119(ed3) III; pmk-3(ok169) IV; wgIs90[nhr-6::TY1::EGFP::3xFLAG + unc-119 (+)], FQ2484 wzEx608[Prab-3::nhr-6 RNAi; Ppes-10::2xglb34AETS-5::dsRed], FQ2486 wzEx609[Pflp-17::nhr-6 RNAi; Ppes10::2xglb-34LETS-5::dsRed], FQ402 ynIs64[Pflp-17::gfp] I, FQ2480 ynIs64[Pflp-17::gfp] I; nhr-6(lg6001) III, MT17370 nIs242 [Pgcy-33::gfp] III; lin-15AB(n765) X, FQ2619 nIs242[Pgcy-33::gfp] nhr-6(wz208) III; lin-15AB(n765) X, CX9683 gcy-31(ok296) X; kyEx2116/gcy-31::SL2::GFP; odr-1::DsRed2], FQ2516 nhr-6 (lg6001) III; gcy-31(ok296) X; kyEx2116 [gcy-31::SL2::GFP; odr-1:: DsRed2], FQ2479 nhr-6(1g6001) III; wzIs113[Pgcy-9::gfp], FQ424 wzIs113[Pgcy-9::gfp], RB1559 acr-2(ok1887) X, CZ9676 acr-2 (n2595 n2420) X, VC20634 acs-22(gk364606) X, CB7468 acs-22 (gk373989) X, RB1292 aex-3(ok1391) X, VC2525 apt-9(ok3247) X, VC2631 B0252.1(gk1114) II, VC40843 B0252.5(gk842840) II, VC1892 BHLHB9(gk3171) IV, VC2695 mapk-15(gk1234) III, VC1880 C06E2.1(ok2451) X, VC40613 C32D5.4(gk722063) II, RB888 casy-1(ok739) II, WU209 cdf-1(n2527) X, LJ1 ceh-37 (ok272) X, LJ2 ceh-37(ok642) X, FQ1885 ceh-54(wz174) X, RB1538 cgp-1(ok1846) V, VC40589 clec-96(gk707996) I, PY1589 cmk-1(oy21) IV, CZ1455 cnd-1(ju29) III, VC40107 cyp-33C7 (gk465749) V, VC1383 $d g k-5(g k 631)$ II, NQ915 dmsr-1(qn45) V, VC20315 dmsr-8(gk245570) V, VC40069 dmsr-8(gk245571) V, VC1487 dyf-11(gk675) X, VC1295 egg-5(ok1781) I, VC40394 egl2(gk616800) V, MT1444 egl-2(n693) V, MT1241 egl-21(n611) IV, MT1218 egl-3(n588) V, MT150 egl-3(n150) V, VC10129 egrh-3 (gk311797) IV, TG1663 ercc-1(tm1981) I, DA1426 exp-2 (sa26ad1426) V, VC40411 F01D5.7(gk623646) II, VC40527 F08G2.5(gk679647) II, VC1240 F22D6.2(ok1685) I, VC20352 F28E10.1(gk200429) IV, VC40449 F59A6.3(gk643207) II, VC2285 F59D12.1(gk1122) X, VC2156 F59D12.1(gk1000) X, VC40427 fil-1(gk633096) X, MT15933 flp-17(n4894) IV, VC378 fut-1(gk183) II, RB756 gar-2(ok520) III, IK427 gcy-23(ni37) IV, FQ1811 glb-25(wz157) V, FQ1545 glb-34(wz139) IV, VC2879 glb-3(ok3630) V/nT1 [qIs51] (IV;V), FX03795 glb-6(tm3795) V, FX07612 glb-7(tm7612) IV, RB1069 gly-12(ok1026) X, MT2426 goa-1(n1134) I, RB2391 grl-29(ok3261) V, FX02620 guk-1 (tm2620) I/hT2 (I;III), VC118 haf-7(gk46) V, ZG31 hif-1(ia4) V, PD4605 hlh-1(cc561) II, JK4099 hlh-2(tm1768) I, RB747 hlh-10 (ok516) V, RB2177 hlh-11(ok2944) III, OH9330 hlh-3(ot354) II; otIs173 [F25B3.3::DsRed2 + ttx-3promB::GFP] III, VC1220 hlh25(ok1710) II, RB1337 hlh-26(ok1453) II, VC475 hsp-16.2(gk249) V, FX01093 hsp-16.41(tm1093) V, VC40856 inpp-1(gk850109)
IV, RB2621 ist-1(ok2706) X, VC40560 ist-1(gk693957) X, VC40322 ist-1(gk573585) X, VC20318 jip-1(gk133506) II, KU2 $j k k-1(\mathrm{~km} 2) \mathrm{X}, \mathrm{VC} 8 \mathrm{jnk}-1(\mathrm{gk} 7) \mathrm{IV}, \mathrm{VC} 20097$ K02F6.8(gk314287) II, DG3393 kin-1(ok338) I; tnEx109 [kin-1(+)+sur-5::GFP], VC3123 kin-21(gk3184) IV, VC40551 klu-2(gk689066) I, RB1619 lfi-1(ok1992) X, VC20694 lgc-30(gk375382) IV, MT14678 lgc-40 (n4545) X, VC40637 lron-6(gk736335) I, RB1037 M04F3.4 (ok981) I, VC2651 mfsd-6(ok3373) III, RB1588 mxl-3(ok1947) X, RB1770 nac-3(ok2274) III, VC40817 nac-3(gk828464) III, RB1059 nhr-3(ok1008) X, VC496 nhr-3(gk258) X, VC1120 nhr-17(gk509) X, VL484 nhr-45(tm1307) X, VC1349 nhr-47(gk954) V, RB844 nhr-50(ok671) V, RB1015 nhr-66(ok940) IV, VC40427 nhr-71 (gk633096) X, FX01033 nhr-88(tm1033) II, VC20665 nhr-88 (gk137345) II, VC1588 nhr-138(gk1018) IV, VC20521 npr-26 (gk337141) IV, VC40742 npr-26(gk789178) IV, PT709 nphp-4 (tm925) him-5(e1490) V, RB1429 npr-15(ok1626) III, SG1 nrx-1 (ds1) V, CX2304 odr-2(n2145) V, VC40505 osta-3(gk669141) II, RB1013 pax-2(ok935) IV, RB2470 pde-6(ok3410) I, LSC39 pdfr-1 (1st34) III, CX14295 pdfr-1(ok3425) III, VC282 pdl-1(gk157) II, RB1972 pgal-1(ok2600) I, RB1721 pghm-1(ok2189) I, RB1709 plp-2(ok2153) X, IK130 pkc-1(nj3) V, VC40710 pqn-44 (gk772270) I, VC20292 pqn-44(gk111052) I, RB809 ptl-1(ok621) III, RB2563 qui-1(ok3571) IV, FQ2326 R11G1.1(wz201) X, VC40538 R11G1.1(gk593575) X, PY1539 ref-1(oy40) II, OH7061

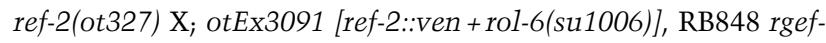
1(ok675) V, LX160 rgs-2(vs17) X, RB1780 rgs-3(ok2288) II, VC40039 rgs-3(gk447133) X, FQ2052 rgs-6(wz186) X, LX533 rgs6(vs62) X, VC20360 rgs-6(gk281200) X, VC20732 rgs-6 (gk386682) X, VC30127 rgs-6(gk963311) X, RM956 ric-4 (md1088) V, CX5156 sad-1(ky289) X, VS22 saeg-1(hj12) V, MT16426 set-9(n4949) IV, RB1053 sgt-1(ok1000) II, RB503 sng-1 (ok234) X, RB737 snt-4(ok503) I, RB2405 sprr-2(ok3290) X, MT19372 sptf-3(n4850) I; nIs283 [gcy-10p::GFP +lin-15(+)] X, CZ1893 syd-1(ju82) II, VC20169 T09F5.10(gk255286) V, RB567 tag-97(ok284) X, PR675 tax-6(p675) IV, RB1835 tbc-18(ok2374) X, VC20591 TMEM104(gk293801) X, VC223 tom-1(ok285) I, VC40235 twk-25(gk527784) IV, CB102 unc-10(e102) X, MT1205 unc-103(n575) III, MT2635 unc-103(e1597 n1213) III, MT13950 unc-103(n4328 n575) III, CB268 unc-41(e268) V, CB408 unc-43 (e408) IV, CZ3391 vab-3(ju468) X, JT366 vhp-1(sa366) II, VC40672 vhp-1(gk756590) II, SJ17 xbp-1(zc12) III; zcIs4[hsp-4:: GFP] V, VC40436 Y105C5A.15(gk636857) IV, VC30162 Y105C5A.15(gk427642) IV, RB1037 Y53G8AR.10(ok981) I, and CB6921 ZK596.1(e2737) IV.

ChIP-seq and data processing

ChIP-seq was performed essentially as previously described (Kranz et al. 2013), using anti-GFP antibodies (Abcam ab290). Chromatin immunoprecipitates were prepared from animals expressing ETS-5::GFP and, in parallel, from nontransgenic animals. Paired-end reads were aligned to the C. elegans genome (WS220) using Bowtie2 (Langmead and Salzberg 2012). Peak calling and genome-wide coverage estimation was obtained with MACS version 2.1.1 (Zhang et al. 2008) using mapped reads from ChIP and input. Peak calling was done for each replicate, and only peaks specific to immunoprecipitates from ETS-5:: GFP transgenics that were detected in all three replicates were considered for further analysis. ChIP-seq signals for each experiment were computed as the difference between coverage detected in chromatin precipitated from ETS-5::GFP transgenics and 
coverage detected in chromatin precipitated from nontransgenic controls. Deeptools (Ramírez et al. 2016) was used to compute enrichment of genomic regions in ETS-5 immunoprecipitates and to generate a heat map of binding near gene transcriptional start sites. Coverage histograms were visualized with the Integrative Genomics Viewer (Thorvaldsdottir et al. 2013).

\section{Cell culture and FACS for RNA-seq}

Embryonic cell cultures for RNA-seq were prepared from wildtype and ets-5(tm1734) mutant animals expressing the BAG cell-specific and ets-5-independent marker wzIs135[P $P_{\text {del } 1: G F P]}$ as previously described (Christensen et al. 2002; Zhang et al. 2002; Fox et al. 2005). Two biological replicates were prepared for each cell type. In brief, embryos were isolated from synchronized populations of hermaphrodites that were treated with hypochlorite, and dissociated into single cells by chitinase treatment. Cells were resuspended in L-15 medium supplemented with $10 \%$ FBS (Sigma) and antibiotics and passed through a $5-\mu \mathrm{m}$ syringe filter (Millipore). Cells were plated onto poly-D-lysine coated single-well chambered cover glasses (Lab-Tek II) and incubated overnight at $25^{\circ} \mathrm{C}$.

GFP-labeled BAG neurons were isolated $\sim 24 \mathrm{~h}$ after dissociation using fluorescence-activated cell sorting (FACS). Dead cells were marked with propidium-iodide and excluded from sorted cells. Sorting was performed on a FACSAria Ilu SORP cell sorter using a $70-\mu \mathrm{m}$ nozzle. Cells were sorted directly into RNA extraction buffer $(10,000$ cells $/ 100 \mu \mathrm{L}$ of buffer) and RNA was purified using the Arcturus PicoPure RNA isolation kit (Thermo Fisher). RNA integrity and concentration were evaluated using an Agilent Bioanalyzer.

RNA-seq analysis of wild-type and ets-5 mutant BAG neurons

cDNA libraries were prepared from RNA (1-2 ng) using the Ovation RNA-seq system V2 (NuGEN), multiplexed, and sequenced as 100 -bp paired-end reads using the Illumina HiSeq 2500 platform. Reads were aligned to the Caenorhabditis elegans genome and transcriptome (Wormbase WS243) using the STAR software package (Dobin et al. 2013). Gene expression quantification was performed using HTSeq (Anders 2015) and differential expression analysis was done using the DeSeq2 package (Anders et al. 2013) in R. Mean read coverage was computed using Deeptools (Ramírez et al. 2016) and coverage histograms were visualized with the Integrative Genomics Viewer (Thorvaldsdottir et al. 2013).

\section{Confocal microscopy}

Young adults were mounted on a $2 \%$ agarose pad made in M9 medium and anaesthetized with $30 \mathrm{mM}$ sodium azide. $Z$ stacks were acquired with a Zeiss LSM700 laser-scanning confocal microscope, and maximum projection images were created using ImageJ (http://imagej.nih.gov/ij). For display purposes, some micrographs were cropped and mounted on a rectangular background colored to match the background of the micrograph.

\section{$\mathrm{CO}_{2}$ avoidance assays}

Avoidance assays were performed essentially as previously described (Martínez-Velázquez and Ringstad 2018). A total of 20 30 adult hermaphrodites was placed on an unseeded $10-\mathrm{cm}$ NGM plate. A custom-made chamber (Supplemental Fig. S6) was pressed into the NGM media, trapping the animals in the chamber. Gas mixes were introduced at $1.5 \mathrm{~mL} / \mathrm{min}$ using a syringe pump (New Era, Inc.). After $35 \mathrm{~min}$, animals were scored as being either on the side with the $\mathrm{CO}_{2}$ inlet or on the side with the air inlet.

\section{Calcium imaging}

Calcium imaging was performed essentially as previously described (Hallem et al. 2011b). Young adults were immobilized with cyanoacrylate veterinary glue (Surgi-Lock, Meridian Animal Health) on a cover glass coated with a $2 \%$ agarose pad made with 10 mM HEPES (pH 7.4). The cover glass was affixed to a custommade air chamber. The specimen was illuminated with 435-nm excitation light and imaged using a $20 \times$ Nikon long-working distance objective ( 0.8 numerical aperture). The emission image was passed through a DV2 image splitter (Photometrics), and the CFP and YFP emission images were projected onto two halves of a cooled CCD camera (Andor). Images were acquired at $10 \mathrm{~Hz}$, with exposure times between 10 and $50 \mathrm{msec}$. Gas perfusion was controlled by three-way valves (Numatics) driven by a custom-made valve controller unit. Custom certified gas mixes used for imaging were obtained from Airgas. Excitation light, image acquisition, and hardware control were performed by the Live Acquisition software package (FEI/Till Photonics). Postacquisition analysis of ratio plots was performed using Matlab (Mathworks) scripts that subtracted linear baseline drift from traces and then applied a five-frame boxcar filter to the time series.

\section{Statistical analyses}

Statistical tests were performed using GraphPad Prism. $P$-values from comparisons of GFP expression levels between BAG neurons from different mutants were made using a Mann-Whitney test and corrected for multiple comparisons. $P$-values from comparisons of the numbers of cells expressing fate markers were made using Fisher's exact test.

\section{Acknowledgments}

We thank the Ringstad laboratory, Jeremy Dasen, Esteban Mazzoni, and Jessica Treisman for helpful comments and stimulating discussions. DNA libraries were prepared by the Genome Technology Core at New York University Medical Center, and we thank Adriana Heguy and Yutong Tang for their expertise and assistance. Strains were provided by the Caenorhabditis Genetics Center, which is funded by the National Institutes of Health (NIH) Office of Research Infrastructure Programs supported by grant P40OD010440. This work was supported by NIH grant R35 GM122573 and a Hirschl Weill-Caulier Foundation career development award (to N.R.). M.R. received support from NIH training grants T32GM007308 and T32HD007520, and NIH grant F30HD094483.

Author contributions: M.R. performed all experiments. M.R. and N.R. designed experiments, analyzed data, and prepared the manuscript.

\section{References}

Anders S, McCarthy DI, Chen Y, Okoniewski M, Smyth GK, Huber W, Robinson MD. 2013. Count-based differential expression analysis of RNA sequencing data using R and Bioconductor. Nat Protoc 8: 1765-1786. doi:10.1038/nprot.2013.099

Anders S, Pyl PT, Huber W. 2015. HTSeq—a Python framework to work with high-throughput sequencing data. Bioinformatics 31: 166-169. doi:10.1093/bioinformatics/btu638 
Bhattacharya A, Aghayeva U, Berghoff EG, Hobert O. 2019. Plasticity of the electrical connectome of C. elegans. Cell 176: 1174-1189.e16. doi:10.1016/j.cell.2018.12.024

Brandt JP, Ringstad N. 2015. Toll-like receptor signaling promotes development and function of sensory neurons required for a C. elegans pathogen-avoidance behavior. Curr Biol 25: 2228-2237. doi:10.1016/j.cub.2015.07.037

Brandt JP, Aziz-Zaman S, Juozaityte V, Martinez-Velazquez LA, Petersen JG, Pocock R, Ringstad N. 2012. A single gene target of an ETS-family transcription factor determines neuronal $\mathrm{CO}_{2}$-chemosensitivity. PLoS One 7: e34014. doi:10.1371/jour nal.pone.0034014

Brandt JP, Rossillo M, Du Z, Ichikawa D, Barnes K, Chen A, Noyes M, Bao Z, Ringstad N. 2019. Lineage context switches the function of a C. elegans Pax6 homolog in determining a neuronal fate. Development 146: dev168153. doi:10.1242/ dev. 168153

Bretscher AJ, Busch KE, de Bono M. 2008. A carbon dioxide avoidance behavior is integrated with responses to ambient oxygen and food in Caenorhabditis elegans. Proc Nat1 Acad Sci 105: 8044-8049. doi:10.1073/pnas.0707607105

Busch KE, Laurent P, Soltesz Z, Murphy RJ, Faivre O, Hedwig B, Thomas M, Smith HL, de Bono M. 2012. Tonic signaling from $\mathrm{O}_{2}$ sensors sets neural circuit activity and behavioral state. Nat Neurosci 15: 581-591. doi:10.1038/nn.3061

Chou JH, Bargmann CI, Sengupta P. 2001. The Caenorhabditis elegans odr-2 gene encodes a novel Ly-6-related protein required for olfaction. Genetics 157: 211-224.

Christensen M, Estevez A, Yin X, Fox R, Morrison R, McDonnell M, Gleason C, Miller DM, Strange K. 2002. primary culture system for functional analysis of C. elegans neurons and muscle cells. Neuron 33: 503-514. doi:10.1016/S0896-6273(02) 00591-3

Dobin A, Davis CA, Schlesinger F, Drenkow J, Zaleski C, Jha S, Batut P, Chaisson M, Gingeras TR. 2013. STAR: ultrafast universal RNA-seq aligner. Bioinformatics 29: 15-21. doi:10 .1093/bioinformatics/bts635

Dosumu-Johnson RT, Cocoran AE, Chang Y, Nattie E, Dymecki SM. 2018. Acute perturbation of Pet1-neuron activity in neonatal mice impairs cardiorespiratory homeostatic recovery. Elife 7: e37857.

Erickson JT, Shafer G, Rossetti MD, Wilson CG, Deneris ES. 2007. Arrest of $5 \mathrm{HT}$ neuron differentiation delays respiratory maturation and impairs neonatal homeostatic responses to environmental challenges. Respir Physiol Neurobiol 159: 85-101. doi:10.1016/j.resp.2007.06.002

Evans RM, Mangelsdorf DJ. 2014. Nuclear receptors, RXR, and the big bang. Cell 157: 255-266. doi:10.1016/j.cell.2014.03 .012

Fox RM, Von Stetina SE, Barlow SJ, Shaffer C, Olszewski KL, Moore JH, Dupuy D, Vidal M, Miller DM 3rd. 2005. A gene expression fingerprint of $C$. elegans embryonic motor neurons. BMC Genomics 6: 42. doi:10.1186/1471-2164-6-42

Gissendanner CR, Kelley K, Nguyen TQ, Hoener MC, Sluder AE, Maina CV. 2008. The Caenorhabditis elegans NR4A nuclear receptor is required for spermatheca morphogenesis. Dev Biol 313: 767-786. doi:10.1016/j.ydbio.2007.11.014

Gissendanner CR, Cardin D, DuBose CJ, El Sayed M, Harmson JS, Praslicka B, Rowan BG. 2014. C. elegans nuclear receptor NHR-6 functionally interacts with the jun-1 transcription factor during spermatheca development. Genesis 52: 29-38. doi: $10.1002 /$ dvg. 22723

Glover JC, Renaud JS, Rijli FM. 2006. Retinoic acid and hindbrain patterning. J Neurobiol 66: 705-725. doi:10.1002/neu.20272
Gramstrup Petersen J, Rojo Romanos T, Juozaityte V, Redo Riveiro A, Hums I, Traunmüller L, Zimmer M, Pocock R. 2013. EGL-13/SoxD specifies distinct $\mathrm{O}_{2}$ and $\mathrm{CO}_{2}$ sensory neuron fates in Caenorhabditis elegans. PLoS Genet 9: e1003511. doi:10.1371/journal.pgen.1003511

Gray JM, Karow DS, Lu H, Chang AJ, Chang JS, Ellis RE, Marletta MA, Bargmann CI. 2004. Oxygen sensation and social feeding mediated by a C. elegans guanylate cyclase homologue. $\mathrm{Na}$ ture 430: 317-322.

Gross E, Soltesz Z, Oda S, Zelmanovich V, Abergel Z, de Bono M. 2014. GLOBIN-5-dependent O2 responses are regulated by PDL-1/PrBP that targets prenylated soluble guanylate cyclases to dendritic endings. I Neurosci Nurs 34: 16726-16738. doi:10.1523/JNEUROSCI.5368-13.2014

Guillermin ML, Castelletto ML, Hallem EA. 2011. Differentiation of carbon dioxide-sensing neurons in Caenorhabditis elegans requires the ETS-5 transcription factor. Genetics 189: 1327-1339. doi:10.1534/genetics.111.133835

Guillermin ML, Carrillo MA, Hallem EA. 2017. A single set of interneurons drives opposite behaviors in C. elegans. Curr Biol 27: 2630-2639.e6. doi:10.1016/j.cub.2017.07.023

Guyenet PG, Bayliss DA. 2015. Neural control of breathing and CO2 homeostasis. Neuron 87: 946-961. doi:10.1016/j.neuron .2015 .08 .001

Hallem EA, Sternberg PW. 2008. Acute carbon dioxide avoidance in Caenorhabditis elegans. Proc Natl Acad Sci 105: 80388043. doi:10.1073/pnas.0707469105

Hallem EA, Dillman AR, Hong AV, Zhang Y, Yano JM, DeMarco SF, Sternberg PW. 2011a. A sensory code for host seeking in parasitic nematodes. Curr Biol 21: 377-383. doi:10.1016/j .cub.2011.01.048

Hallem EA, Spencer WC, McWhirter RD, Zeller G, Henz SR, Rätsch G, Miller DM III, Horvitz HR, Sternberg PW, Ringstad N. 2011 b. Receptor-type guanylate cyclase is required for carbon dioxide sensation by Caenorhabditis elegans. Proc Natl Acad Sci 108: 254-259. doi:10.1073/pnas.1017354108

He S, Philbrook A, McWhirter R, Gabel CV, Taub DG, Carter MH, Hanna IM, Francis MM, Miller DM 3rd. 2015. Transcriptional control of synaptic remodeling through regulated expression of an immunoglobulin superfamily protein. Curr Biol 25: 2541-2548. doi:10.1016/j.cub.2015.08.022

Heard M, Maina CV, Morehead BE, Hoener MC, Nguyen TQ, Williams CC, Rowan BG, Gissendanner CR. 2010. A functional NR4A nuclear receptor DNA-binding domain is required for organ development in Caenorhabditis elegans. Genesis 48: 485-491. doi:10.1002/dvg.20646

Hendricks T, Francis N, Fyodorov D, Deneris ES. 1999. The ETS domain factor Pet-1 is an early and precise marker of central serotonin neurons and interacts with a conserved element in serotonergic genes. J Neurosci Nurs 19: 10348-10356. doi:10 .1523/JNEUROSCI.19-23-10348.1999

Hoekstra EJ, von Oerthel L, van der Heide LP, Kouwenhoven WM, Veenvliet JV, Wever I, Jin YR, Yoon JK, van der Linden AJ, Holstege FC, et al. 2013. Lmxla encodes a rostral set of mesodiencephalic dopaminergic neurons marked by the Wnt/B-catenin signaling activator R-spondin 2. PLoS One 8: e74049. doi:10.1371/journal.pone.0074049

Horowitz LB, Brandt JP, Ringstad N. 2019. Repression of an activity-dependent autocrine insulin signal is required for sensory neuron development in C. elegans. Development 146: dev182873. doi:10.1242/dev.182873

Hu JS, Vogt D, Lindtner S, Sandberg M, Silberberg SN, Rubenstein JLR. 2017. Coup-TF1 and Coup-TF2 control subtype and laminar identity of MGE-derived neocortical interneurons. Development 144: 2837-2851. doi:10.1242/dev.150664 
Kranz AL, Jiao CY, Winterkorn LH, Albritton SE, Kramer M, Ercan S. 2013. Genome-wide analysis of condensin binding in Caenorhabditis elegans. Genome Biol 14: R112. doi:10 .1186/gb-2013-14-10-r112

Kratsios P, Pinan-Lucarré B, Kerk SY, Weinreb A, Bessereau JL, Hobert O. 2015. Transcriptional coordination of synaptogenesis and neurotransmitter signaling. Curr Biol 25: 1282-1295. doi:10.1016/j.cub.2015.03.028

Langmead B, Salzberg SL. 2012. Fast gapped-read alignment with Bowtie 2. Nat Methods 9: 357-359. doi:10.1038/nmeth.1923

Ma DK, Ringstad N. 2012. The neurobiology of sensing respiratory gases for the control of animal behavior. Front Biol 7: 246253. doi:10.1007/s11515-012-1219-x

Machanick P, Bailey TL. 2011. MEME-ChIP: motif analysis of large DNA datasets. Bioinformatics 27: 1696-1697. doi:10 .1093/bioinformatics/btr189

Martínez-Velázquez LA, Ringstad N. 2018. Antagonistic regulation of trafficking to Caenorhabditis elegans sensory cilia by a retinal degeneration 3 homolog and retromer. Proc Natl Acad Sci 115: E438-E447. doi:10.1073/pnas.1712302115

Miller-Fleming TW, Petersen SC, Manning L, Matthewman C, Gornet M, Beers A, Hori S, Mitani S, Bianchi L, Richmond J, et al. 2016. The DEG/ENaC cation channel protein UNC-8 drives activity-dependent synapse removal in remodeling GABAergic neurons. Elife 5: e14599. doi:10.7554/eLife.14599

Milward K, Busch KE, Murphy RJ, de Bono M, Olofsson B. 2011. Neuronal and molecular substrates for optimal foraging in Caenorhabditis elegans. Proc Natl Acad Sci 108: 2067220677. doi:10.1073/pnas.1106134109

Petersen SC, Watson JD, Richmond JE, Sarov M, Walthall WW, Miller DM 3rd. 2011. A transcriptional program promotes remodeling of GABAergic synapses in Caenorhabditis elegans. I Neurosci Nurs 31: 15362-15375. doi:10.1523/JNEUROSCI .3181-11.2011

Pönniö T, Conneely OM. 2004. nor-1 regulates hippocampal axon guidance, pyramidal cell survival, and seizure susceptibility. Mol Cell Biol 24: 9070-9078. doi:10.1128/MCB.24.20.90709078.2004

Praslicka B, Gissendanner CR. 2015. The C. elegans NR4A nuclear receptor gene $n h r-6$ promotes cell cycle progression in the spermatheca lineage. Dev Dyn 244: 417-430. doi:10.1002/ dvdy. 24244

Ramírez F, Ryan DP, Grüning B, Bhardwaj V, Kilpert F, Richter AS, Heyne S, Dündar F, Manke T. 2016. Deeptools2: a next generation web server for deep-sequencing data analysis. $\mathrm{Nu}$ cleic Acids Res 44: W160-W165. doi:10.1093/nar/gkw257

Ray RS, Corcoran AE, Brust RD, Kim JC, Richerson GB, Nattie E, Dymecki SM. 2011. Impaired respiratory and body temperature control upon acute serotonergic neuron inhibition. Science 333: 637-642. doi:10.1126/science. 1205295

Ray RS, Corcoran AE, Brust RD, Soriano LP, Nattie EE, Dymecki SM. 2013. Egr2-neurons control the adult respiratory response to hypercapnia. Brain Res 1511: 115-125. doi:10.1016/j .brainres.2012.12.017

Rengarajan S, Yankura KA, Guillermin ML, Fung W, Hallem EA. 2019. Feeding state sculpts a circuit for sensory valence in Caenorhabditis elegans. Proc Natl Acad Sci 116: 17761781. doi:10.1073/pnas.1807454116
Romanos TR, Petersen JG, Riveiro AR, Pocock R. 2015. A novel role for the zinc-finger transcription factor EGL-46 in the differentiation of gas-sensing neurons in Caenorhabditis elegans. Genetics 199: 157-163. doi:10.1534/genetics.114.172049

Smith ES, Martinez-Velazquez L, Ringstad N. 2013. A chemoreceptor that detects molecular carbon dioxide. I Biol Chem 288: 37071-37081. doi:10.1074/jbc.M113.517367

Taubert S, Ward JD, Yamamoto KR. 2011. Nuclear hormone receptors in nematodes: evolution and function. Mol Cell Endocrinol 334: 49-55. doi:10.1016/j.mce.2010.04.021

Thorvaldsdottir H, Robinson JT, Mesirov JP. 2013. Integrative genomics viewer (IGV): high-performance genomics data visualization and exploration. Brief Bioinform 14: 178-192. doi:10 $.1093 / \mathrm{bib} / \mathrm{bbs} 017$

Walthall WW, Plunkett JA. 1995. Genetic transformation of the synaptic pattern of a motoneuron class in Caenorhabditis elegans. J Neurosci 15: 1035-1043. doi:10.1523/JNEUROSCI.1502-01035.1995

Wilson L, Gale E, Chambers D, Maden M. 2004. Retinoic acid and the control of dorsoventral patterning in the avian spinal cord. Dev Biol 269: 433-446. doi:10.1016/j.ydbio.2004.01.034

Wyler SC, Spencer WC, Green NH, Rood BD, Crawford L, Craige C, Gresch P, McMahon DG, Beck SG, Deneris E. 2016. Pet-1 switches transcriptional targets postnatally to regulate maturation of serotonin neuron excitability. J Neurosci 36: 17581774. doi:10.1523/JNEUROSCI.3798-15.2016

Zetterström RH, Solomin L, Jansson L, Hoffer BJ, Olson L, Perlmann T. 1997. Dopamine neuron agenesis in Nurr1-deficient mice. Science 276: 248-250. doi:10.1126/science. 276.5310 .248

Zhan Y, Du X, Chen H, Liu J, Zhao B, Huang D, Li G, Xu Q, Zhang M, Weimer BC, et al. 2008. Cytosporone B is an agonist for nuclear orphan receptor Nur77. Nat Chem Biol 4: 548-556. doi:10.1038/nchembio.106

Zhang Y, Ma C, Delohery T, Nasipak B, Foat BC, Bounoutas A, Bussemaker HJ, Kim SK, Chalfie M. 2002. Identification of genes expressed in C. elegans touch receptor neurons. Nature 418: 331-335. doi:10.1038/nature00891

Zhang Y, Liu T, Meyer CA, Eeckhoute J, Johnson DS, Bernstein BE, Nussbaum C, Myers RM, Brown M, Li W, et al. 2008. Model-based analysis of ChIP-seq (MACS). Genome Biol 9: R137. doi:10.1186/gb-2008-9-9-r137

Zhang L, Le W, Xie W, Dani JA. 2012. Age-related changes in dopamine signaling in Nurrl deficient mice as a model of Parkinson's disease. Neurobiol Aging 33: 1001.e7-1001.e16. doi:10 .1016/j.neurobiolaging.2011.03.022

Zhou HM, Walthall WW. 1998. UNC-55, an orphan nuclear hormone receptor, orchestrates synaptic specificity among two classes of motor neurons in Caenorhabditis elegans. J Neurosci 18: 10438-10444. doi:10.1523/JNEUROSCI.18-24-10438 .1998

Zimmer M, Gray JM, Pokala N, Chang AJ, Karow DS, Marletta MA, Hudson ML, Morton DB, Chronis N, Bargmann CI. 2009. Neurons detect increases and decreases in oxygen levels using distinct guanylate cyclases. Neuron 61: 865-879. doi:10 $.1016 /$ j.neuron.2009.02.013 


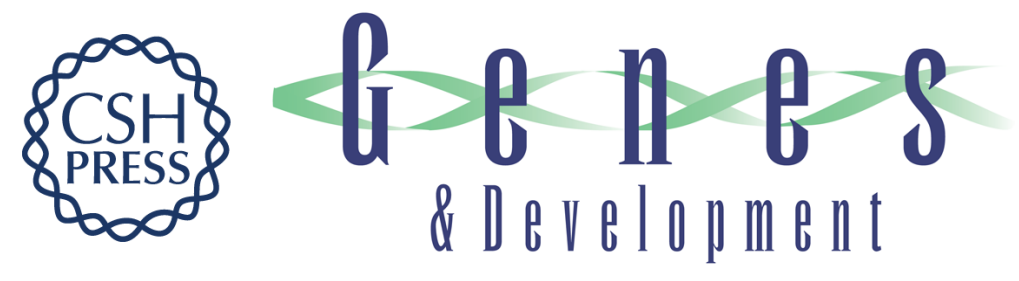

\section{Development of specialized sensory neurons engages a nuclear receptor required for functional plasticity}

Mary Rossillo and Niels Ringstad

Genes Dev. 2020, 34: originally published online November 12, 2020

Access the most recent version at doi:10.1101/gad.342212.120

\section{Supplemental http://genesdev.cshlp.org/content/suppl/2020/11/11/gad.342212.120.DC1 Material}

References This article cites 62 articles, 22 of which can be accessed free at:

http://genesdev.cshlp.org/content/34/23-24/1666.full.html\#ref-list-1

Creative This article is distributed exclusively by Cold Spring Harbor Laboratory Press for the first

Commons six months after the full-issue publication date (see

License http://genesdev.cshlp.org/site/misc/terms.xhtml). After six months, it is available under a Creative Commons License (Attribution-NonCommercial 4.0 International), as described at http://creativecommons.org/licenses/by-nc/4.0/.

Email Alerting Receive free email alerts when new articles cite this article - sign up in the box at the top Service right corner of the article or click here.

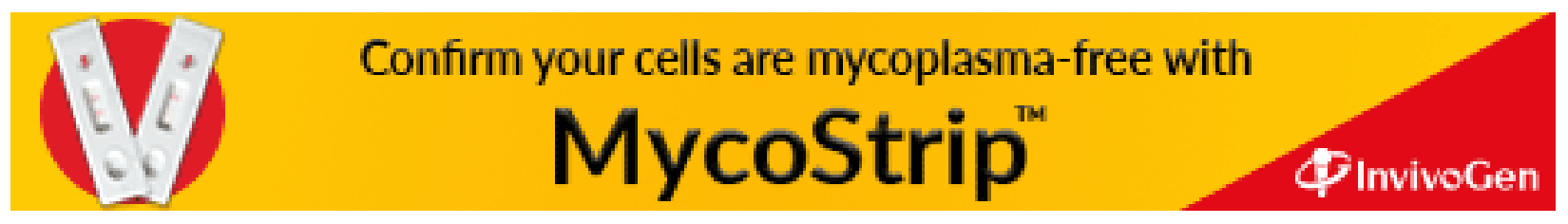

